\title{
Predictive Shrinkage Error Model for Selective Laser Sintering Hybrid Milling*
}

\author{
Trung Nghiep NGUYEN ${ }^{* *}$, Hideki AOYAMA ${ }^{* * *}$ and Ichiro ARAIE ${ }^{\dagger}$
}

\begin{abstract}
Selective laser sintering hybrid milling (SHM) is an advanced manufacturing technology that combines the flexibility of additive manufacturing with the precision of traditional machining. In SHM, instead of machining the products after they are completely formed using selective laser sintering (SLS), the milling process is conducted alternatively with the SLS. This allows direct fabrication of extremely complicated and high-precision products on the same workstation. However, shrinkage owing to SLS is a fundamental problem of this technology, because it creates significant dimensional errors in the final product. In this study, a method is proposed to calculate shrinkage error in SHM based on equations built from the data collected using FEA. Consequently, the calculation result is used to compensate the SHM shrinkage error. The experimental results of this study confirm that the error prediction model and the proposed compensation technique work efficiently in reducing SHM error.
\end{abstract}

Key words: SLS hybrid milling; shrinkage error; compensation

\section{Introduction}

Selective laser sintering (SLS), a common additive manufacturing technology, has many promising applications. Selective laser sintering for metal powder, especially, has enormous potential in industry because it allows the fabrication of complex products that traditional machining cannot achieve ${ }^{1)}$. However, poor surface quality, dimensional and geometry errors are the fundamental problems of this technology ${ }^{2), 3)}$. Products fabricated from metal powder using SLS are usually in the form of raw products, and need to be machined again with computer numerical controller (CNC) milling. To solve this problem, a new technology called selective laser sintering hybrid milling (SHM) was invented ${ }^{32,4)}$, whereby after a block of $n_{l}$ layers (about 10 to 25 ) is deposited using SLS, it is immediately machined. With this innovation, complex and high-quality surfaces can be created directly on the same workstation.

An SHM-based machine is actually a combination of an SLS and a high-speed milling machine. Recently, this type of machine has been introduced to the market. The basic schematic diagram of the SHM forming process is illustrated in Fig. 1. The product includes the base plate and the part. First, $n_{l}$ layers are continuously deposited on top of the base plate using laser sintering. After that, the sintering process is interrupted, and the first $n_{l}$ layers are machined using CNC milling. Similarly, the next $n_{l}$ layers are deposited using laser sintering and then machined. This hybrid laser sintering and milling process is repeated until all part layers of are formed.

In SHM technology, to avoid smelting metal drop on the finished face, a new milling technique called step machining was invented (Patent No.

\footnotetext{
* Received on February 12, 2020

Accepted on May 22, 2020

* Student member, Keio University (3-14-1 Hiyoshi, Kohoku-ku, Yokohama, Kanagawa 223-8522, JAPAN)

*** Full member, Keio University (3-14-1 Hiyoshi, Kohoku-ku, Yokohama, Kanagawa 223-8522, JAPAN)

${ }^{\dagger}$ Full member, Sodick Co., Ltd (Ka-11, Miya-Machi, Kaga-shi, Ishikawa 922-0595, JAPAN)
}

$3687672)^{5}$. Figure 2 illustrates the step machining process after the layers of the third block have just deposited. First, Block 3 (the third block) is rough machined (usually with a ball end milling tool). After that, the machine is changed to a T-shape end milling tool for finishing milling in Block 2 . With the step machining process, smelting metal only drops on the semi-finished faces, and is removed in the next milling finish step.

Although SHM is a promising technique, it also has a fundamental problem deformation due to material shrinkage. Figure 3 illustrates the deformation due to material shrinkage in SHM. A new layer is deposited using laser sintering with a temperature over the smelting point (approximately $1500^{\circ} \mathrm{C}$ ). The temperature of the new layer quickly drops because the layer thickness is extremely thin. During the cooling process, the new layer shrinks and pressures the material under it. There are hundreds to thousands of layers in a product; thus, shrinkage is repeated as many times as there are layers. Therefore, the effect of shrinkage in SHM is complicated and significant.

Some studies investigated the effect of geometrical deformation owing to shrinkage in SLS and selective laser melting (SLM), such as in 6)8). However, no previous literature has reported on dimensional errors owing to shrinkage on the machined face. In SLS and SLM, products are machined after all layers are formed by laser sintering; therefore, shrinkage does not affect the finished faces. However, in the SHM technology, as explained in Fig. 1, milling is conducted during the sintering process; therefore, shrinkage directly affects the finished faces. The deformation caused by shrinkage becomes the dimensional error of the final product and significantly reduces the accuracy.

In this study, the dimensional error owing to shrinkage is termed shrinkage error, which was first investigated in the previous study 9). In that study, finite element analysis (FEA) was used to calculate the shrinkage error in SHM, and an experiment was conducted to confirm the FEA results. After that, a compensation technique was proposed based on the calculated shrinkage error to improve the SHM accuracy. Although the proposed method gave a promising result, it is limited in actual production due to the extremely long simulation time ( 3 to 4 days for a simulation of a $60 \mathrm{~mm} \times 20 \mathrm{~mm} \times 10 \mathrm{~mm}$ sample). In this study, a new method is 


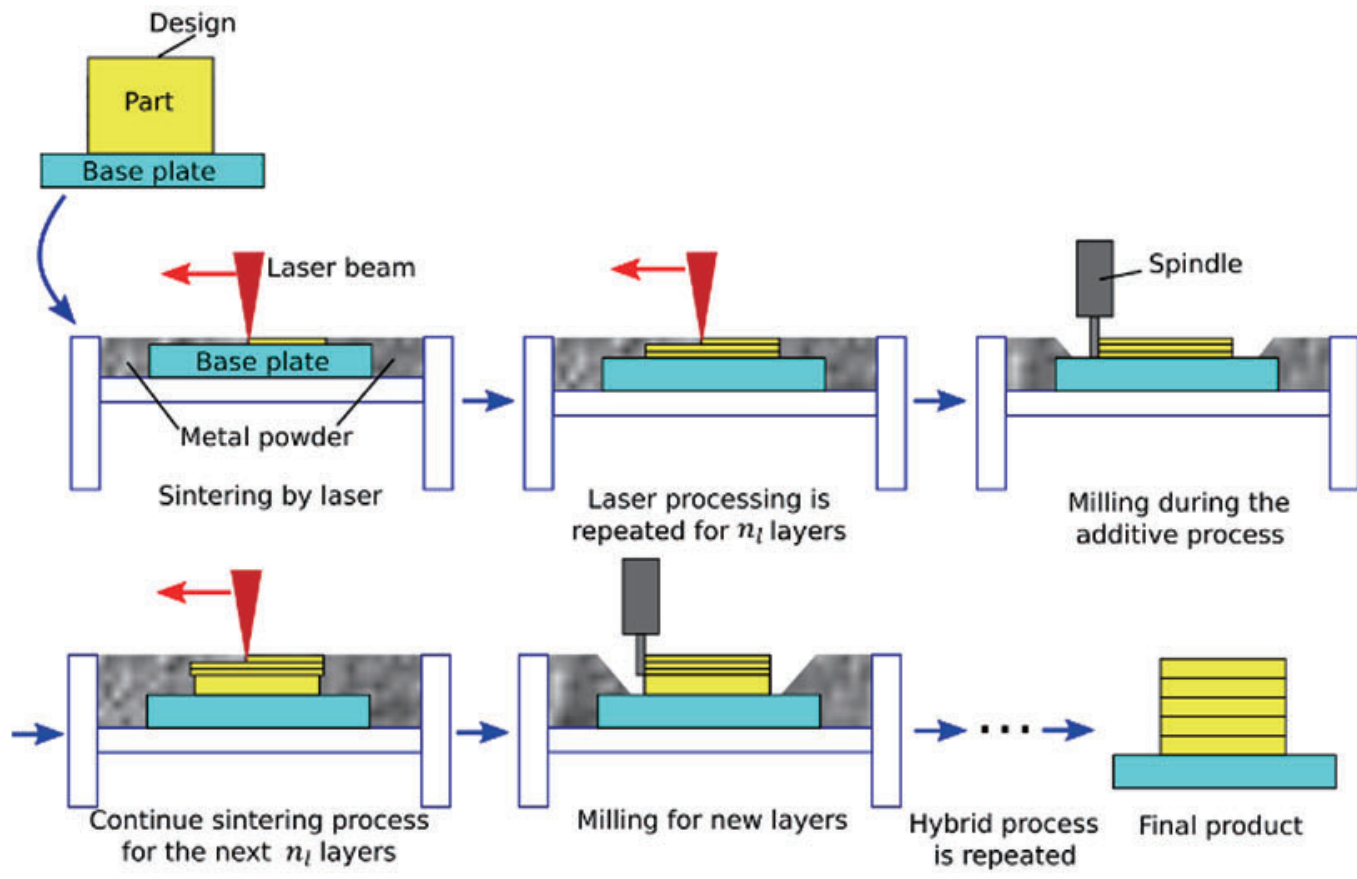

Fig. 1 Selective laser sintering hybrid milling process schematic diagram

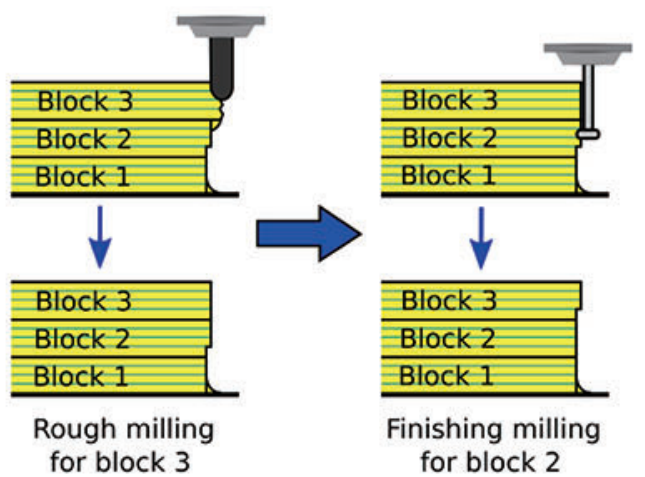

Fig. 2 Step machining in SHM

proposed to derive the equations that predict shrinkage error. This method is expected to calculate shrinkage error within a short period, and is intended to be applied in the actual production.

The objective of this study is building a mathematical model for SHM shrinkage error based on the shrinkage error data collected using FEA. From the previous study results ${ }^{9}$, the shrinkage error components and shrinkage mechanism are understood. In this study, the experience equations are proposed to calculate the shrinkage error components. Next, an interpolation technique is used to determine the parameters of the proposed equations. The data used to interpolate the equation parameters of the equations are collected using the FEA model that was developed in the previous research ${ }^{9}$. After successfully building the equations to calculate shrinkage error based on the FEA data, the effectiveness of these equations is experimentally confirmed.

\section{Process of shrinkage error evolution in SHM}

As mentioned in Section 1, dimensional errors owing to shrinkage (shrinkage error) in the finished machined face only appear in SHM. The mechanism of shrinkage error was first reported in our previous study 9), wherein three shrinkage rules and unclamping effects were proposed to

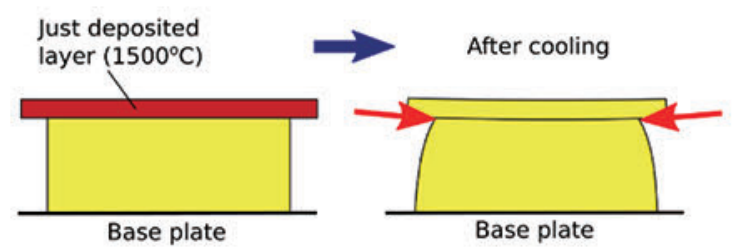

Fig. 3 Effect of shrinkage in cooling process

explain the evolution process of shrinkage error in SHM. This explanation was confirmed using FEA and experimental results. Based on that explanation, a mathematical model for shrinkage error is developed in this study. First, in this section, the shrinkage error evolution process in SHM is presented.

2.1 Shrinkage error mechanism (the first and second shrinkage rules)

Figure 4 illustrates the effect due to sintering process shrinkage in one block of layers. First, a new layer is deposited on top of the part and begins to cool. After cooling, the top of the part is pressed and deformed. This process is repeated for $n_{l}$ layers in the block and creates the deformation effect illustrated in Fig. 4. In the previous study, the first and second shrinkage rules were proposed to describe this phenomenon. The first shrinkage rule said that the area which is closest to the shrinking layer is more strongly affected by shrinkage. The second shrinkage rule said that the area far enough from the shrinking layer is insignificantly affected by shrinkage.

With label $\mathrm{P}$ as one point on the wall of the part as illustrated in Fig. 4, and $h_{s p}$ is the distance from $\mathrm{P}$ to the top of the part. In other words, $h_{s p}$ is the distance from $P$ to the shrinking layers. Based on the first and second shrinkage rules, when $h_{s p}$ increases, the shrinkage error at $\mathrm{P}$ will be reduced; moreover, if $h_{s p}$ is large enough, the shrinkage error at point $\mathrm{P}$ is near zero. This mechanism was confirmed in the previous study ${ }^{9}$.

2.2 Evolution of shrinkage error in SHM (the third shrinkage rule)

As explained in Section 1 with the illustrations in Fig. 1 and Fig. 2, the process of SHM includes the repeated hybrid sintering and step milling process. The process of shrinkage error evolution is based on this forming 


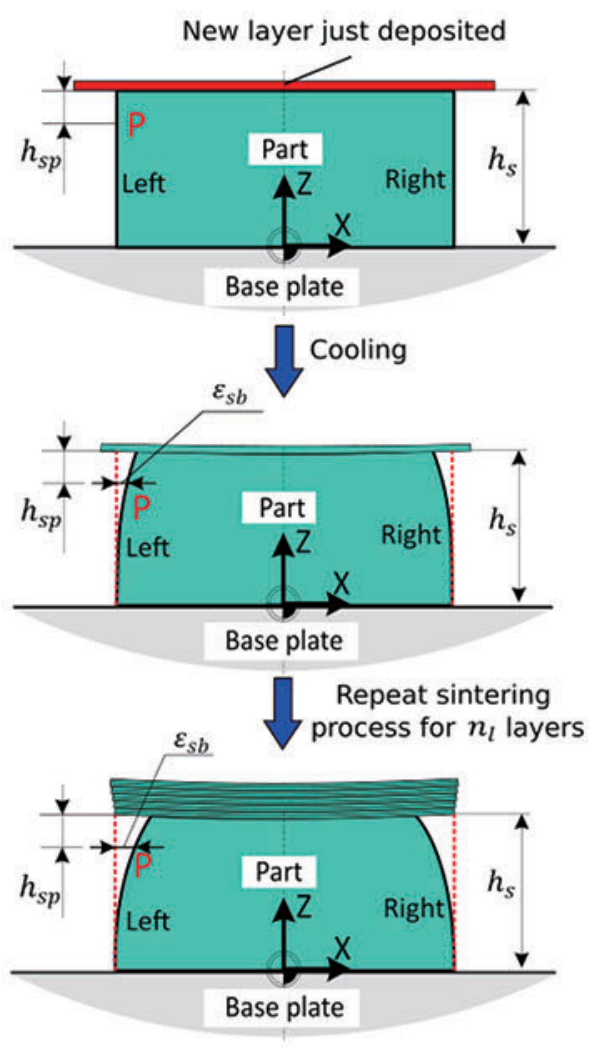

Fig. 4 Mechanism of shrinkage

process, and is illustrated in Fig. 5. After sintering and milling two blocks, the shrinkage error did not yet appear in the part. Next, layers of Block 3 are deposited and cause deformation in Blocks 1 and 2. Subsequently, step milling is used to rough machine Block 3 and finish machine Block 2. This milling process eliminates the current deformation in block 2 , but the deformation in Block 1 still remains and becomes the first shrinkage error component which is denoted by $\varepsilon_{s b 3 \_}$. The process of deformation happens similarly when Block 4 is formed as illustrated in Fig. 5 . Shrinkage of Block 4 creates more deformation in Block 1, and this increased shrinkage error is denoted by $\varepsilon_{s b 4 \_1}$. The shrinkage error in Block 1 at this stage includes two components as illustrated in Fig. 5 . The process is iterated until $\mathrm{n}$ blocks are formed. During this process, after one more block is deposited, the shrinkage errors in the lower blocks continuously increase. For example, after the $\mathrm{n}^{\text {th }}$ block, the shrinkage error of Block 1 includes $\varepsilon_{s b 3_{1} 1}, \varepsilon_{s b 4_{-} 1}, \ldots, \varepsilon_{s b(n-1)_{-} 1}, \varepsilon_{s b n_{-} 1}$ as illustrated in Fig. 5. The process of shrinkage error evolution explained above is identified as the third shrinkage rule ${ }^{9}$. Generally, $e_{s j}$ is the shrinkage error of block $j$ after $\mathrm{n}$ blocks have been formed, and is termed the shrinkage error component due to the shrinkage of blocks above. Based on the third shrinkage rule, $e_{s j}$ is given by Equation (1):

$$
e_{s j}=\sum_{i=j+2}^{n} \varepsilon_{s b i j},
$$

where $j$ is the position of the block from which shrinkage error is calculated, $\varepsilon_{s b i_{-} j}$ is the shrinkage error increasing in block $j$ owing to the shrinkage of block $i$. With $j$ from 1 to $n-2, e_{s j}$ is calculated using Equation (1); $e_{s(n-1)}$ and $e_{s n}$ equal zero.

After all $\mathrm{n}$ blocks are formed, the height of the sample theoretically reaches the requirement. However, in the real process, 10 extra layers are deposited to guarantee that the height of the part is over the requirement.

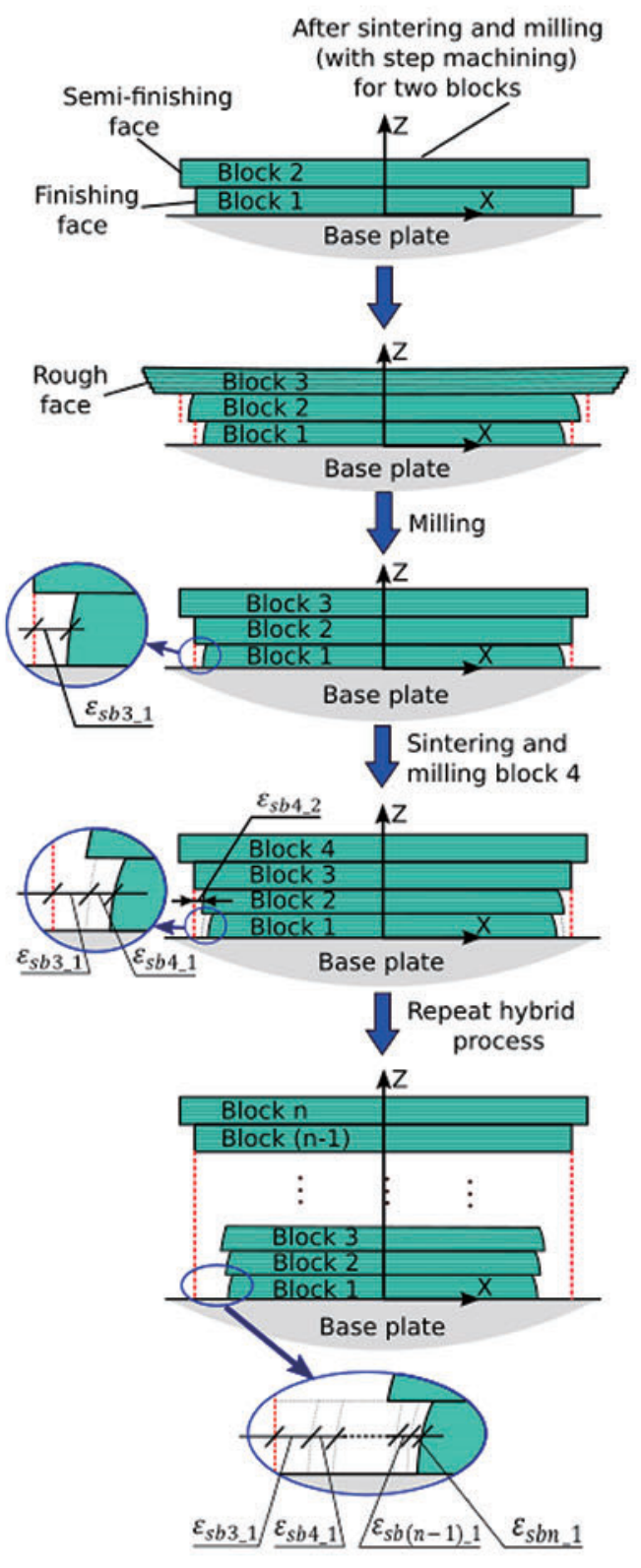

Fig. 5 Mechanism of shrinkage

Subsequently, the extra layers are machined to finish the top face of the sample in the final step of the forming process. The shrinkage mechanism in the extra layers is similar to the shrinkage mechanism in one block. However, the shrinkage error owing to the extra layers is less than that of one block because the number of layers is less. The error owing to shrinkage of extra layers to the under blocks underneath also follows the first and second shrinkage rule, and is denoted by $e_{e x}$.

\subsection{Effect of unclamping to shrinkage error}

After finishing the forming process, the sample is released from the machine table. This step is called unclamping. Unclamping itself does not create shrinkage; however, the residual stress in the base plate bends it after being released, which affects the part and introduces more error. This error is named unclamping error and is denoted by $e_{u c}$. The mechanism of the base plate bending is explained as follows. The shrinkage during the forming process of the layers creates compressive stress on the top of the base plate. In contrast, for balancing the internal force, the bottom of the 
base plate develops tensile stress. With the residual stress as explained above, the base plate tends to bend into the part. Figure 6 (a) illustrates the effect of bending on unclamping (Fig. 6 considers only the unclamping error). Based on the model in Fig. 6 (a), the part sample also bends due to the base plate bending effect. The top of the part is most strongly deformed, and the deformation continuously reduces from the top to the bottom of the part. However, from the results of our previous study ${ }^{9}$, it was observed that deformation owing to unclamping at the bottom of the part tends to be enhanced. This phenomenon is called boundary enhancing and is illustrated in Fig. 6 (b). Boundary enhancing supposedly occurs due to the sudden change in the dimension from the length of the base plate to the length of the part. In general, the deformation owing to unclamping is the combination of both the bending effect and the boundary enhancing effect as illustrated in Fig. 6 (c).

\subsection{The final shrinkage error in SHM}

The final shrinkage error includes three components: shrinkage error owing to the shrinkage in other blocks $\left(e_{s j}\right)$, shrinkage error owing to shrinkage in the extra layers $\left(e_{e x}\right)$, and unclamping error $\left(e_{u c}\right)$. The $e_{s j}$ is the first and most important component. The $e_{e x}$ is the smallest component which is created after $e_{s j}$ when the extra layers are added onto the top. Finally, $e_{u c}$ is created when the base plate is released. The distribution of the three shrinkage error components is determined based on the shrinkage error evolution in SHM that was investigated in the previous study ${ }^{9}$. Shrinkage error of a point $\mathrm{P}$ in block $\mathrm{j}\left(E_{s j}\right)$ is given by Equation (2) as follows:

$$
E_{s j}=e_{s j}+e_{e x}+e_{u c},
$$

The shrinkage error on the part wall in SHM has a saw-tooth shape as illustrated in Fig. 7 because the top of the block is strongly pressed and deforms more than the bottom as explained by the first shrinkage rule. Shrinkage errors at the lower blocks are larger than the higher blocks because shrinkage is repeated many more times at the lower blocks than at the higher blocks as explained in the third shrinkage rule. However, the shrinkage error at the bottom blocks tends to become stable and does not increase more because when the distance from a block to the new sintered layer is large enough, the shrinkage effect is almost zero as explained in the second shrinkage rule.

\section{Building equations for shrinkage error in SHM}

Section 2 presents the three components of shrinkage error in SHM that were investigated and confirmed in the previous study ${ }^{9}$. To determine the shrinkage error, the three error components in Equation (2) must be determined. In the previous research "), FEA was used to determine SHM shrinkage error. However, as mentioned in Section 1, the limitation of this method is time-consuming; therefore, is not suitable for actual production. In this study, a mathematical model is proposed to be used to calculate shrinkage error with the aim of being applicable to actual production. The process of building equations for the three shrinkage error components in SHM is illustrated in Fig. 8. Based on the shrinkage rules presented in Section 2, potential equations are predicted for the shrinkage error components. Subsequently, shrinkage error data is collected using an FEA model, then it is used to interpolate the equation parameters. Consequently, the result of the equations is tested with experimentation. If the result

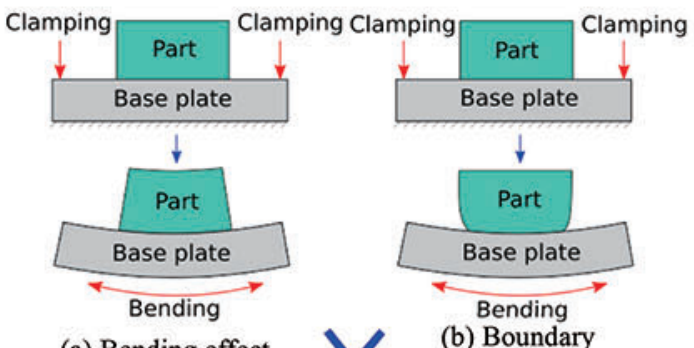

(a) Bending effect

(b) Boundary

enhancing effect

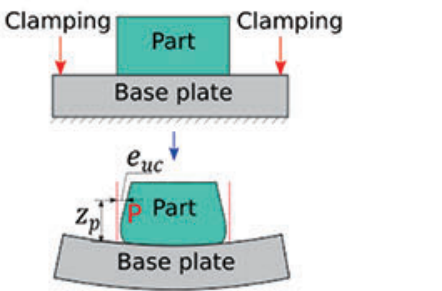

(c) Combination of two effects

Fig. 6 Effect of unclamping to shrinkage error

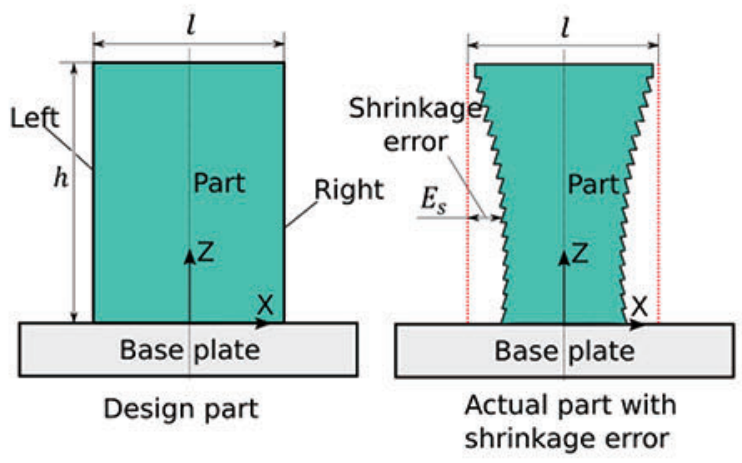

Fig. 7 Final shrinkage error shape in SHM

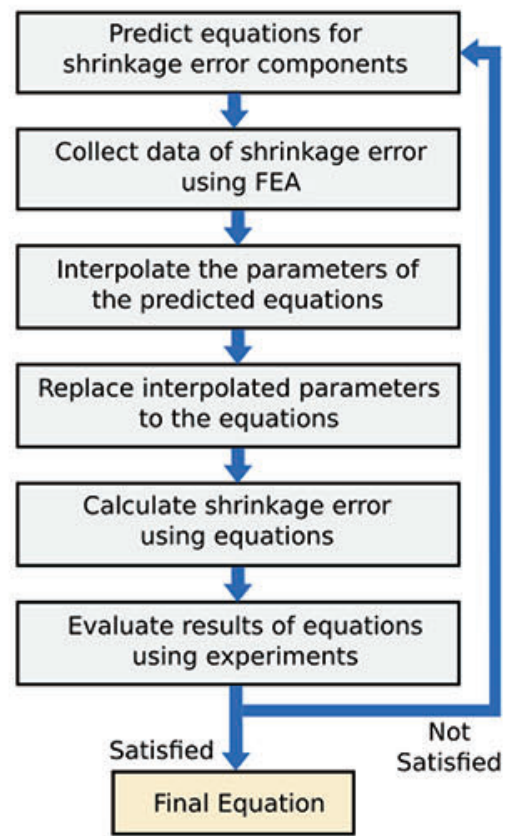

Fig. 8 Process of building equations for shrinkage error in SHM

satisfies the accuracy requirement, the proposed equations are confirmed, if not, the above process is repeated by modifying the predicted equations. In this study, the expected calculation accuracy is $0.01 \mathrm{~mm}$. This section 
presents the final equations that are optimized to satisfy the expected accuracy and shorten calculation time, Section 4 presents the interpolation process for the equation parameters, and Section 5 presents confirmation using experimentation.

\subsection{Model description}

The equations in this study are developed to calculate shrinkage error for a rectangular shape as illustrated in Fig. 9. In this model the $\mathrm{z}$ axis is the symmetric axis. This model uses two input variables: the length $(l)$, and height $(h)$ of the part. The other forming process parameters are considered as forming conditions such as material, base plate dimension, and laser sintering conditions. The model part has $n$ blocks, and each block has $n_{l}$ layers as illustrated in Fig. 9.

The positions in the model that shrinkage errors are calculated are termed calculation points. In this model, shrinkage error is calculated at 11 calculation points for each block. The calculation step (distance between the two nearest calculation points) is $0.1 \mathrm{~mm}$ (Fig. 10). At the boundary between two blocks, there are two calculation points: the bottom point of the above block and the top of the below block. The calculation points of one block is illustrated in Fig. 10. Shrinkage error of each calculation point in the model includes three components as explained in equation (2). Shrinkage error of each point is illustrated in Fig. 11. In the next sections, experience equations used to calculate the components of shrinkage error are presented in detail.

\subsection{Equation for shrinkage error due to the shrinkage in other blocks $\left(e_{s j}\right)$}

The first shrinkage error component is the shrinkage error due to the shrinkage in other blocks to block $j\left(e_{s j}\right)$ that was explained in detail in Section 2.2 and Equation (1). The $e_{s j}$ is the sum of $\varepsilon_{s b i_{-} j}$ (the shrinkage error increasing on block $j$ owing to the shrinkage in block $i$, with $i$ from $j+2$ to $n$ ). The proposed equation for $\varepsilon_{s b i_{j} j}(\mathrm{~mm})$ is given in Equation (3):

$$
\varepsilon_{s b i_{-j}}=\frac{1}{s_{a} h_{s p i_{j}}^{\omega_{e s}}+s_{b}}+s_{c}
$$

where $\omega_{e s}$ is a proposed constant, given in Table $1 ; s_{a}$ and $s_{b}$ are the functions of the length of the sample $(l)$ and are alternatively given by Equations (4) and (5); $s_{c}$ is given by Equation (6), and $h_{s p i_{j} j}(\mathrm{~mm})$ is the distance from the calculation point to the bottom of block $i$, as illustrated in Fig. 12.

$$
\begin{aligned}
& s_{a}=\alpha_{s 1} k_{s a}^{l}+\alpha_{s 2}, \\
& s_{b}=\beta_{s 1} k_{s b}^{l}+\beta_{s 2},
\end{aligned}
$$

where $l(\mathrm{~mm})$ is the length of the sample; $\alpha_{s 1}, \alpha_{s 2}, \beta_{s 1}$, and $\beta_{s 2}$ are the parameters depending on the forming conditions, and $k_{s a}, k_{s b}$ are the proposed constants given in Table 1 . Forming conditions are introduced in Section 3.1, and parameters dependents on forming conditions such as $\alpha_{s 1}, \alpha_{s 2}, \beta_{s 1}$, and $\beta_{s 2}$ are determined using FEA data presented in Section 4 .

$$
s_{c}=\gamma_{s 1} e^{k_{s c} h_{s}}+\gamma_{s 2},
$$

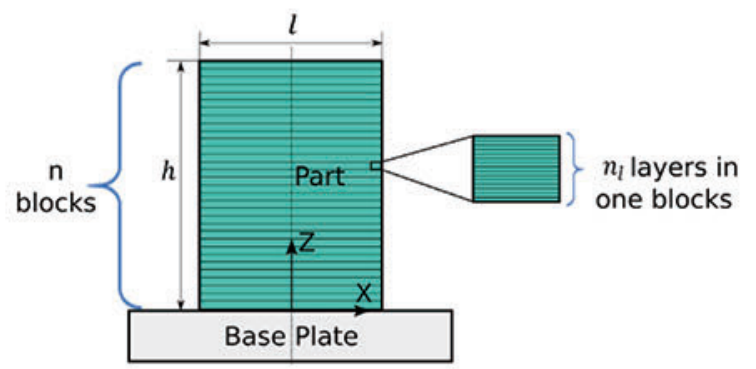

Fig. 9 Number of blocks and layers in the model

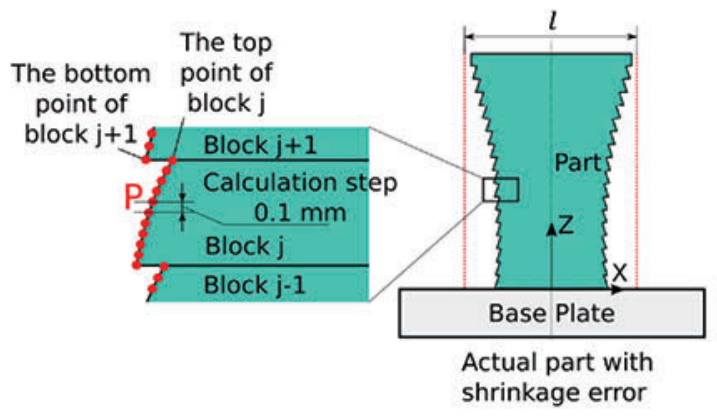

Fig. 10 Calculation points in the model

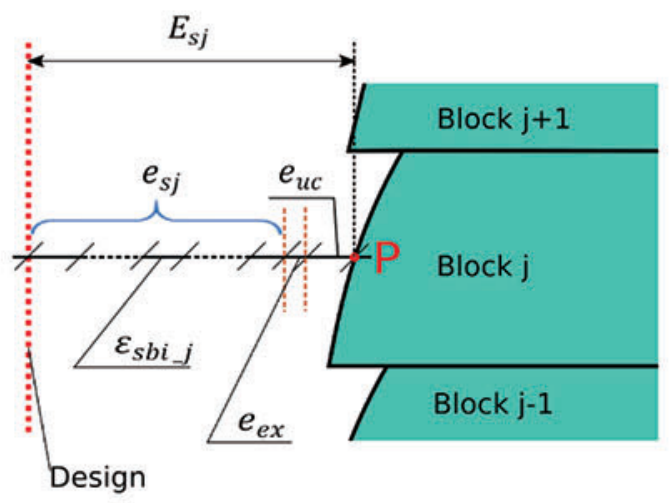

Fig. 11 Components of shrinkage errors in the model

where $k_{s c}$ is a proposed constant, given in Table $1, h_{s}(\mathrm{~mm})$ is the distance from the bottom of block $i$ to the top of the base plate illustrated in Fig. 12; $e$ is the mathematic constant $e$; and $\gamma_{s 1}, \gamma_{s 2}$ are alternatively given by Equations (7) and ( 8 ).

$$
\begin{aligned}
& \gamma_{s 1}=c_{s a 1} k_{s c a}^{l}+c_{s a 2} \\
& \gamma_{s 2}=c_{s b 1} l^{2}+c_{s b 2} l+c_{s b 3}
\end{aligned}
$$

where $c_{s a 1}, c_{s a 2}, c_{s b 1}, c_{s b 2}$, and $c_{s b 3}$ are the parameters depending on the forming conditions; and $k_{s c a}$ is a proposed constant, given in Table 1 .

Equations (4) - (6) are used to calculate the parameters $s_{a}, s_{b}$, and $s_{c}$ of Equation (3). These equations are also derived using trial and error based on experience. First, data of $s_{a}, s_{b}$, and $s_{c}$ are calculated by interpolating the shrinkage error data; the interpolation and data of $s_{a}, s_{b}$, and $s_{c}$ are described and given in Section 4. Then, the potential equations for $s_{a}, s_{b}$, and $s_{c}$ are predicted based on their interpolated data. The initial predicted equations are modified by the experience of researchers until they perfectly matched the data. These best matched equations for data of $s_{a}, s_{b}$ and $s_{c}$ are proposed and presented in the Equations (4)- 


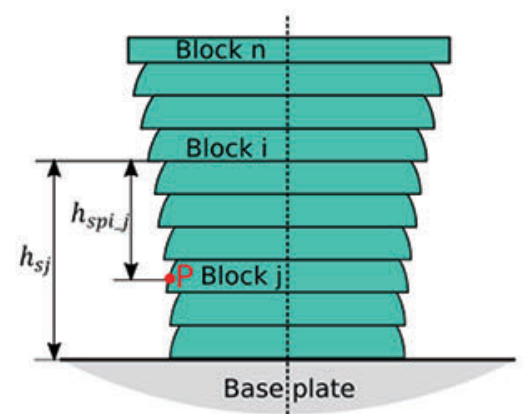

Fig. 12 Parameters to determine $\varepsilon_{s b i_{-} j}$

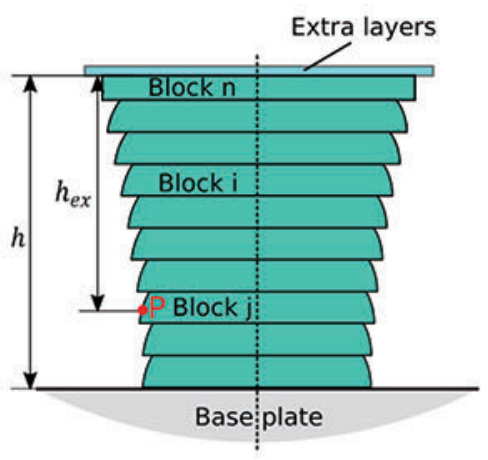

Fig. 13 Parameters to determine $e_{e x}$

(6). Equations (7) and (8) are used to calculate $\gamma_{s 1}$ and $\gamma_{s 2}$ of Equation

(6). The derivatives of these two equations for $\gamma_{s 1}$ and $\gamma_{s 2}$ are similar to $s_{a}, s_{b}$, and $s_{c}$.

\subsection{Equation for shrinkage error due to shrinkage in the extra layers $\left(e_{e x}\right)$}

Shrinkage error due to shrinkage in the extra layers $\left(e_{e x}\right)$ is introduced in Section 2.2. The proposed equation for $e_{e x}$ is,

$$
e_{e x}=\frac{1}{s_{e a} h_{e x}^{\omega_{e x}}+s_{e b}}+s_{e c}
$$

where $\omega_{e x}$ is a proposed constant, given in Table $1 ; h_{e x}(\mathrm{~mm})$ is the distance from the calculation point to the extra layer as illustrated in Fig. 13; $s_{e a}, s_{e b}$, and $s_{e c}$ are the functions of the length of sample $(l)$ and are given by Equations (10), (11), and (12) alternatively. Equations (3) and (9) have the same form because the effect of one block and extra layers are similar. However, shrinkage error caused by extra layers is smaller than one block because the number of extra layers (10) are smaller than one block (25). The derivative of Equation (9) is also similar to Equation (3) which is explained at the beginning of Section 3.

$$
\begin{aligned}
& s_{e a}=\alpha_{e x 1} k_{e a}^{l}+\alpha_{e x 2}, \\
& s_{e b}=\beta_{e x 1} k_{e b}^{l}+\beta_{e x 2}, \\
& s_{e c}=\gamma_{e x 1} l^{2}+\gamma_{e x 2} l+\gamma_{e x 3},
\end{aligned}
$$

where $l(\mathrm{~mm})$ is the length of the sample, $\alpha_{\text {ex } 1}, \alpha_{\text {ex } 2}, \beta_{\text {ex } 1}, \beta_{\text {ex } 2}$, $\gamma_{e x 1}, \gamma_{e x 2}$, and $\gamma_{e x 3}$ are the parameters depending on the forming conditions; and $k_{e a}, k_{e b}$ are the proposed constants, given in Table 1. In this study, block $\mathrm{n}$ is finish machined after depositing the extra layers; therefore, $e_{e x}$ in block $\mathrm{n}$ is zero. Equations (10) - (12) are used to calculate the parameters $s_{e a}, s_{e b}$ and $s_{e c}$ of Equation (9). The derivatives of these equations and the collection of data for $s_{e a}, s_{e b}$, and $s_{e c}$ are similar to $s_{a}, s_{b}$, and $s_{c}$.

\subsection{Equation for unclamping error $\left(e_{u c}\right)$}

Unclamping error $\left(e_{u c}\right)$ is explained in Section 2.3. In this study, the proposed Equation (13) is used to calculate $e_{u c}(\mathrm{~mm})$,

$$
e_{u c}=\frac{1}{s_{u a} z_{p}^{\omega_{u c}}+s_{u b}}+s_{u c} z_{p}+s_{u d}
$$

where $\omega_{e x}$ is a proposed constant, given in Table $1 ; z_{p}(\mathrm{~mm})$ is the distance from the calculated point to the bottom of the part as illustrated in Fig. 6 (c), and $s_{u a}, s_{u b}, s_{u c}$, and $s_{u d}$ are given by Equations (14), (15), (16), and (17), respectively,

$$
\begin{aligned}
& s_{u a}=\alpha_{u c 1} k_{u a}^{l}+\alpha_{u c 2}, \\
& s_{u b}=\beta_{u c 1} l^{2}+\beta_{u c 2} l+\beta_{u c 3}, \\
& s_{u c}=\frac{1}{\gamma_{u c 1} k_{u c}^{l}+\gamma_{u c 2}}+\gamma_{u c 3}, \\
& s_{u d}=\delta_{u c 1} l^{2}+\delta_{u c 2} l+\delta_{u c 3},
\end{aligned}
$$

where $l(\mathrm{~mm})$ is the length of the sample; $\alpha_{u c 1}, \alpha_{u c 2}, \beta_{u c 1}, \beta_{u c 2}$, $\beta_{u c 3}, \gamma_{u c 1}, \gamma_{u c 2}, \gamma_{u c 3}, \delta_{u c 1}, \delta_{u c 2}$, and $\delta_{u c 3}$ are the parameters depending on the forming conditions; and $k_{u a}$ and $k_{u c}$ are the proposed constants, given in Table 1. Equations (14) - (17) are used to calculate the parameters $s_{u a}, s_{u b}, s_{u c}$ and $s_{u d}$ of Equation(13). The derivatives of these equations and the collection of data for $s_{u a}, s_{u b}, s_{u c}$, and $s_{u d}$ are similar to $s_{a}, s_{b}$, and $s_{c}$.

Table 1 Proposed constants

\begin{tabular}{ccc}
\hline $\begin{array}{c}\text { Constants for } \\
\text { calculating } e_{s j}\end{array}$ & $\begin{array}{c}\text { Constants for } \\
\text { calculating } e_{e x}\end{array}$ & $\begin{array}{c}\text { Constants for } \\
\text { calculating } e_{u c}\end{array}$ \\
\hline$\omega_{e s}=0.6$ & $\omega_{e x}=0.42$ & $\omega_{u c}=0.9$ \\
$k_{s a}=0.9397$ & $k_{e a}=0.940$ & $k_{u a}=1.025$ \\
$k_{s b}=0.9554$ & $k_{e b}=0.953$ & $k_{u c}=0.91$ \\
$k_{s c}=-0.3$ & & \\
$k_{s c a}=0.969$ & & \\
\hline
\end{tabular}

The Equations (3) - (17) and the proposed constants for them are derived by trial and error based on experience of researchers. Many potential equations were predicted and tested with the collected data. The equations presented in this paper are the most suitable within the set of predicted equations. These equations and the proposed constants are only confirmed with the specific forming conditions in this study.

The definition processes of Equations (3) - (8) are illustrated in Fig. 14. Equation (3) is the main equation which is derived from the FEA 
results. Equations (4) - (6) are the sub-equations of Equation (3), and are derived from data interpolated from FEA results. Equations (7) and (8) are the sub-equations of Equation (6); data to define these equations are obtained by interpolating data derived by Equations (6). The methods to interpolate data from FEA results are explained in Section 4. Similarly, the definition processes of Equations (9) - (12) are summarized in Fig. 15; the definition processes of Equations (13) - (17) are illustrated in Fig. 16.

The constants in the equations are determined by the same way with the parameters. Let take the process of determining the constant $\omega_{e s}$ in Equation (3) as an example. Equation (3) with four parameters: $s_{a}, s_{b}$, $s_{c}$, and $\omega_{e s}$, was firstly predicted. After that, $\varepsilon_{s b i_{-} j}$ data was used to interpolate these four parameters. The interpolation result shows that $s_{a}$, $s_{b}, s_{c}$ depended on $l$ and $h_{s}$ while $\omega_{e s}$ was nearly unchanged $\left(\omega_{e s}\right.$ $\approx 0.6$ ). Therefore, the constant $\omega_{e s}$ was replaced by 0.6 in Equation (3). The other parameters $s_{a}, s_{b}$, and $s_{c}$ are interpolated again after replacing $\omega_{e s}=0.6$ in Equation (3); the results are given in Fig. 24 and Table 3. The input data and the interpolation processes to determine the constant $\omega_{e s}$ are similar to that of the parameters $s_{a}, s_{b}$, and $s_{c}$. The FEA model used to calculate input data and interpolation processes are explained in detail in Section 4. The other constants in Table 1 are determined by the same way.

\section{Determination of parameters for shrinkage error equations using FEA data}

The next step of developing SHM shrinkage error equations is determining the parameters dependent on forming conditions. In this study, except for the two input parameters of the mathematical model $(l$ and $h$ ), the other forming process parameters are termed forming conditions as introduced in Section 3.1. As presented in Section 3, each equation for the shrinkage error components has parameters dependent on forming conditions. In total, there are 27 parameters dependent on forming conditions. In this study, an interpolation technique is used to determine these parameters. First, the shrinkage error components are calculated using FEA; then, data collected from FEA is used to interpolate the parameters in the corresponding equations. After determining all of 27 parameters, they are replaced in the equations and used to calculate the shrinkage error. The results of the mathematical model are evaluated using experimentation.

The interpolation technique is usually used to estimate a formula for a data set collected from experimentation. However, in the actual SHM forming process, determining the single shrinkage error components is extremely difficult. This is because the current equipment can measure the final shrinkage error only, while the interpolation process needs the data from all shrinkage error components. The forming process explained in Section 1 is the chain of hybrid sintering and milling processes, and after repeating this hybrid process, one more component of shrinkage error appears in the part. The process must be conducted continuously in a closed chamber, therefore, stopping and measuring shrinkage error components during the forming process is impossible. In this study, experiments are only used to evaluate the calculation results of the mathematical model, not to determine the parameters and the constants. Using FEA is the best choice to replace experimentation for collecting shrinkage error data. Moreover, the FEA method was used in our previous study 9) to calculate shrinkage error, and proved that it can give reliable results.

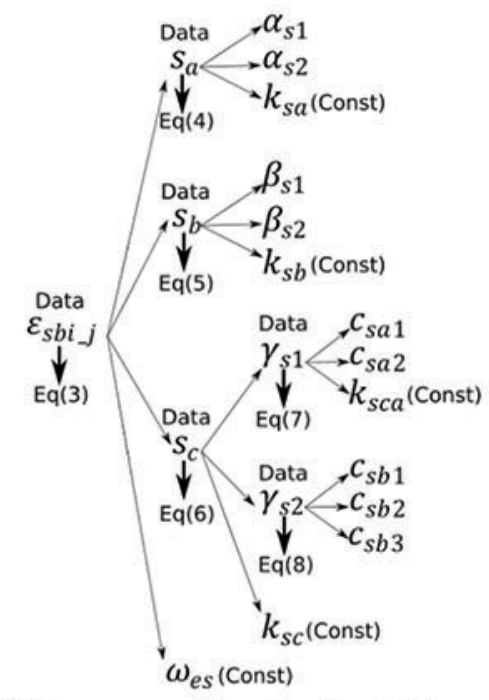

Fig. 14 Definition process of equation for shrinkage error due to the shrinkage in other blocks (Equations (3) - (8))

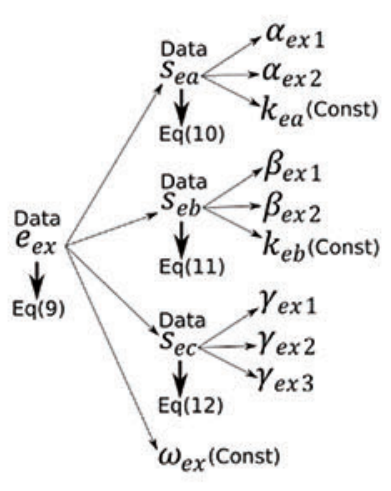

Fig. 15 Definition process of equation for shrinkage error due to the extra layers

(Equations (9) - (12))

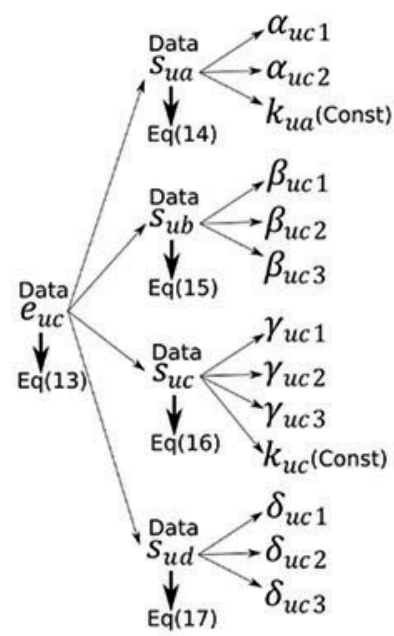

Fig. 16 Definition process of equation for unclamping error (Equations (13) -

\subsection{Testing forming condition}

In this study, the parameters of the shrinkage error equations are determined for the following forming condition provided by Sodick $\mathrm{Co}$., Ltd. This forming condition was also investigated using FEA in the previous study ${ }^{9}$. The sample model is given in Fig. 17 with the origin at the center of the base plate top face. The width of the part $(w)$ is unchanged and equal to $10 \mathrm{~mm}$. The base plate material is $\mathrm{S} 50 \mathrm{C}$ without hardening ${ }^{10)}$ and that of the part is maraging steel ${ }^{11)}$. The thickness of one layer is $0.04 \mathrm{~mm}$, and the number of layers in one block is 25 ; therefore, thickness of one block is $1 \mathrm{~mm}$. In the laser sintering process, a length of approximately $0.2 \mathrm{~mm}$ at both sides of the layers is added for the milling process with $0.035 \mathrm{~mm}$ for finishing milling and approximately $0.165 \mathrm{~mm}$ for rough milling. The milling conditions are given in Table 2 . The processing conditions of the SLS process, including scanning pattern, raster pitch, raster length, laser power, scanning speed, and laser spot diameter, are explained and given in Fig. 18. The wavelength of the laser source is $1070 \mathrm{~nm}$; the intensity of the laser beam has the Gaussian Distribution; the laser transmitter is the Single Mode. The absorption rates 


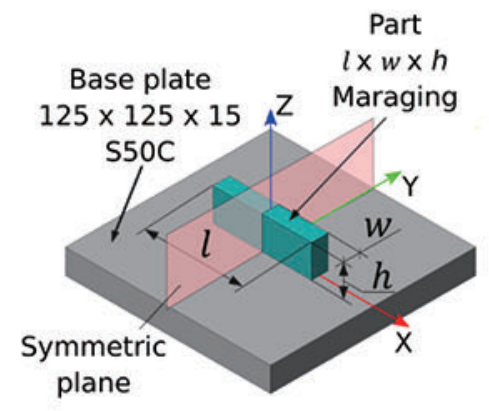

Fig. 17 Material and dimensions of the samples

Table 2 Milling conditions

\begin{tabular}{lccc}
\hline & Rough milling & $\begin{array}{c}\text { Semi-finishing } \\
\text { milling }\end{array}$ & $\begin{array}{c}\text { Finishing } \\
\text { milling }\end{array}$ \\
\hline Tool & Ball end-mill & Ball end-mill & T end-mill \\
\hline Tool radius & $1 \mathrm{~mm}$ & $1 \mathrm{~mm}$ & $0.5 \mathrm{~mm}$ \\
\hline Revolution(rpm) & 17000 & 17000 & 25000 \\
\hline $\begin{array}{l}\text { Feed rate } \\
\text { (mm/min) }\end{array}$ & 1200 & 1200 & 1200 \\
\hline $\begin{array}{l}\text { Radius depth of } \\
\text { cut (mm) }\end{array}$ & $\approx 0.1$ & 0.065 & 0.035 \\
\hline Z-pitch (mm) & 0.08 & 0.08 & 0.05 \\
\hline XY-pitch (mm) & 0.08 & 0.08 & 0.05 \\
\hline
\end{tabular}

of metallic solids depend on the wavelength of light. In the experiment, the wavelength of the laser source is $1070 \mathrm{~nm}$, and the metal power is Maraging steel, i.e., an Fe based material. The absorption rate of laser beam to material is approximately $40 \%$ base on the reference ${ }^{12}$.

\subsection{Collection of shrinkage error data using FEA}

As mentioned above, shrinkage error data used to determine equation parameters are collected using the FEA model which was developed and confirmed in the previous study 9). To collect data for this study, simulations are conducted for six samples that have the length $(l)$ alternatively of $30,40,50,60,70$, and $80 \mathrm{~mm}$, and the height $(h)$ is $20 \mathrm{~mm}$. Similar to the previous study, the simulations are conducted using a two dimensional (2D) FEA model with plane stress analysis. The FEA model is built using Code Aster with the elastoplastic behavior of material ${ }^{13)}$. The simulation process includes $n+2$ steps as illustrated in Fig. 19. The hybrid process of depositing and milling for $n$ blocks are simulated from Step 1 to Step $n$. Because the height of the samples is $20 \mathrm{~mm}$, and the block thickness is $1 \mathrm{~mm}, \mathrm{n}$ is 20. Each step includes the simulation for $n_{l}$ layers ( $n_{l}=25$ in the samples) and a simulation for milling. The simulation for one layer includes thermal and mechanical simulations. The thermal simulation calculates the thermal distribution during the cooling process, and then the result of the thermal simulation is used to calculate the shrinkage deformation in the mechanical simulation. From the results of the shrinkage deformation, shrinkage error components are determined. The detailed explanations of the simulation technique in each FEA step are given in the previous research 9 .

The thermal simulation is importance because its result is the input to calculation deformation. In the thermal simulation, the forming temperature for one layer is $1500^{\circ} \mathrm{C}$, and time for cooling is $30 \mathrm{~s}$. The thermal simulation result of the first layer at $2.8 .10^{-5} \mathrm{~s}$ for the $70 \mathrm{~mm} \times 20$

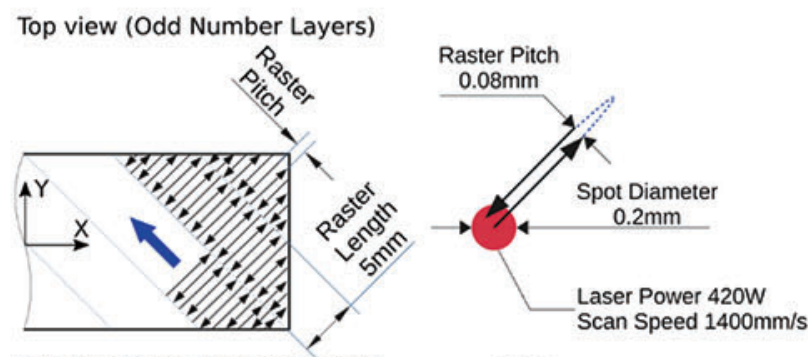

Top view (Even Number Layers)

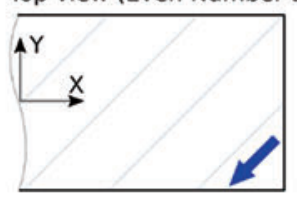

(b) Laser power, scans, spot diameter, raster pitch

(a) Scanning pattern

Fig. 18 Laser sintering conditions

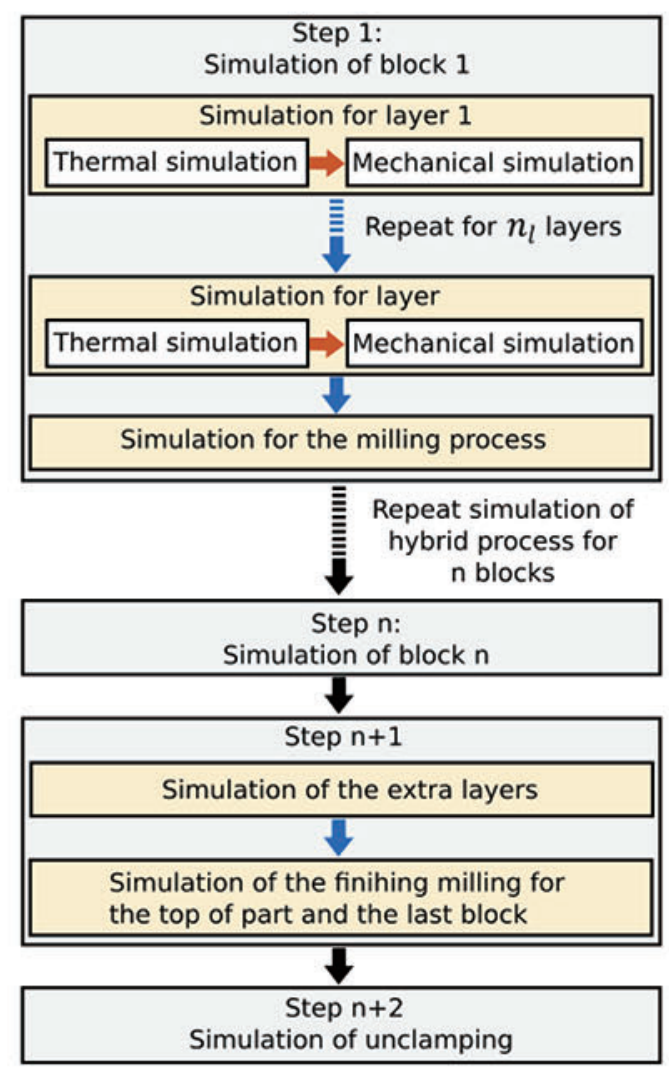

Fig. 19 Simulation process using FEA

mm sample is illustrated in Fig. 20, and the temperature of the middle point of the first layer in $30 \mathrm{~s}$ cooling is illustrated in Fig. 21. The detail explanations of thermal simulation were presented in the previous study ${ }^{9}$.

Figure 19 illustrates the final deformation result in the $\mathrm{X}$ direction in the simulation for the $70 \mathrm{~mm} \times 20 \mathrm{~mm}$ sample. In this result, the deformation of the nodes on the left wall is the shrinkage error $\left(E_{s j}\right)$ that needs to be calculated. The distance between two nodes is $0.1 \mathrm{~mm}$ as illustrated in Fig. 22. The color in Fig. 22 represents for the deformation.

To calculate the shrinkage error component in this simulation, the increase of deformation corresponding with the forming step that creates this shrinkage error component is determined. Assume that the shrinkage error due to the shrinkage of Block 10 is now determined. First, the simulation result is taken for the deformation before Block 10 is sintered 


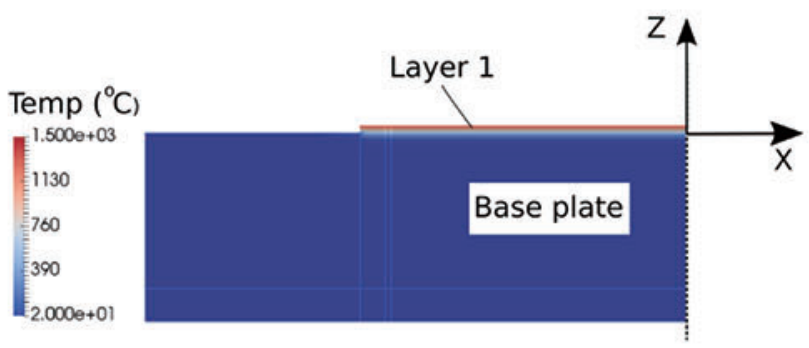

Fig. 20 The thermal simulation result of the first layer at 2.8.10 $s$ for the sample $70 \mathrm{~mm} \times 20 \mathrm{~mm}$

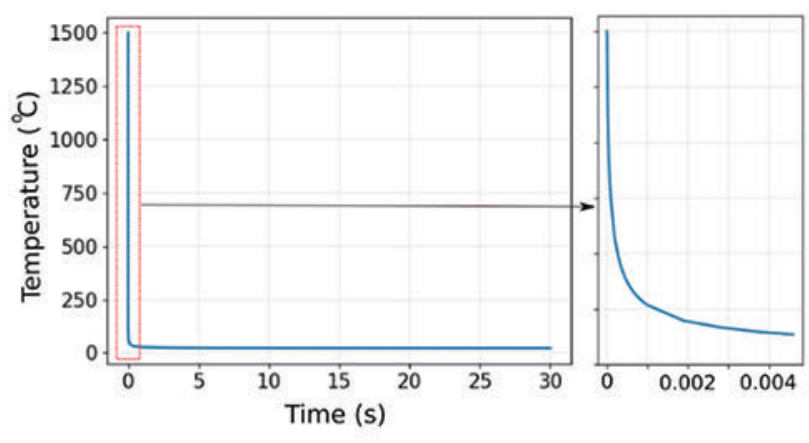

Fig.21 The thermal simulation result of the middle point of the first layer for the sample $70 \mathrm{~mm} \times 20 \mathrm{~mm}$

$\left(E_{s 10_{-} b}\right)$, and after Block 10 is sintered $\left(E_{s 10_{e} e}\right)$ as illustrated in Fig. 23. Consequently, the absolute value is taken for the difference between $E_{s 10_{-} e}$ and $E_{s 10_{-} b}$. This result is the shrinkage error components generated in Step $10\left(\varepsilon_{s b 10_{j} j}\right.$, with $\left.j=1,2, \ldots, 9\right)$. In the proposed mathematical model, this shrinkage error component corresponds with Equation (3), however, in Equation (3), $\varepsilon_{s b i_{j} j}$ is the function of $h_{s p i_{-} j}$ while in Fig. 23, the calculated data is the function of the $\mathrm{Z}$ coordinate. Therefore, before using this data to interpolate the Equation (3) parameters, the $\mathrm{Z}$ coordinate is converted to $h_{s p i_{-} j}$. After conversion, the collected data of $\varepsilon_{s b 10_{-} j}$ is illustrated in Fig. 26 by the blue graph. At the forming step in Figs. 20 and 21, Block 9 has only been rough machined while blocks 1 to 8 have already been finish-machined. The length of Block 9 at this step is longer than Blocks 1 to 8 by $0.035 \mathrm{~mm} \times 2(0.035 \mathrm{~mm}$ is the finish-milling depth of the cut). Therefore, there is a sudden change of value at $Z=8 \mathrm{~mm}$ in Fig. 23. This is considered a calculation error. However, because the finish milling depth of the cut is very small compared with the sample length, this calculation error is insignificant.

\subsection{Interpolation of parameters in the proposed equations using shrinkage error data collected from FEA}

An interpolation technique is commonly used to derive a matched curve for a given data set. Many methods can be used for interpolation such as the least square method. Many software packages are currently available to conduct the interpolation process. In this study, the "curve_fit" function of the "scipy.optimize" library is used to interpolate the parameters of the proposed equations. This is an open source library running on Python; it is free and helps to save calculation time. This function provides some fitting solution methods; "least-squares" method is chosen in this study. The "curve_fit" function details are given in the Reference ${ }^{14)}$. The short instruction of using "scipy.optimize" library to find fitting equations is given in the Reference ${ }^{15}$; the detail explanation can

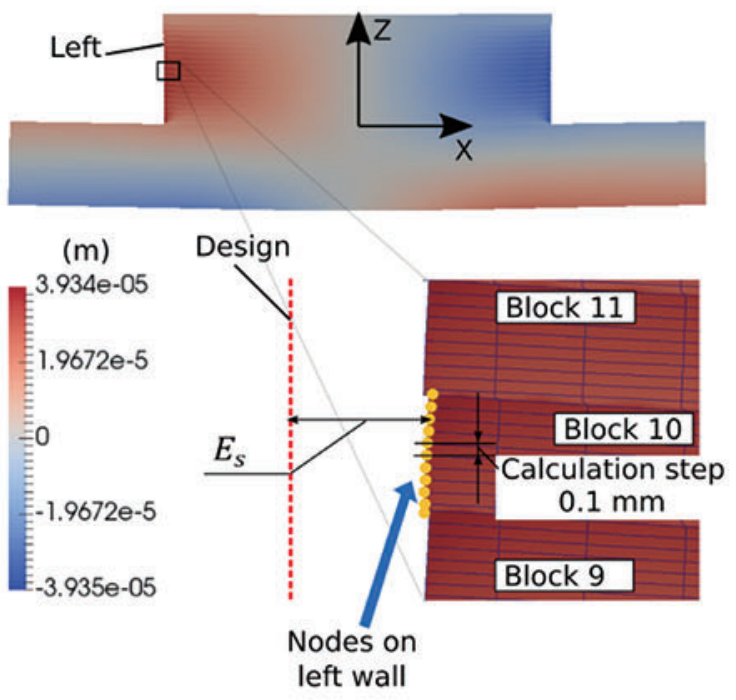

Fig. 22 The final result of simulation for the $70 \mathrm{~mm} \times 20 \mathrm{~mm}$ sample using FEA

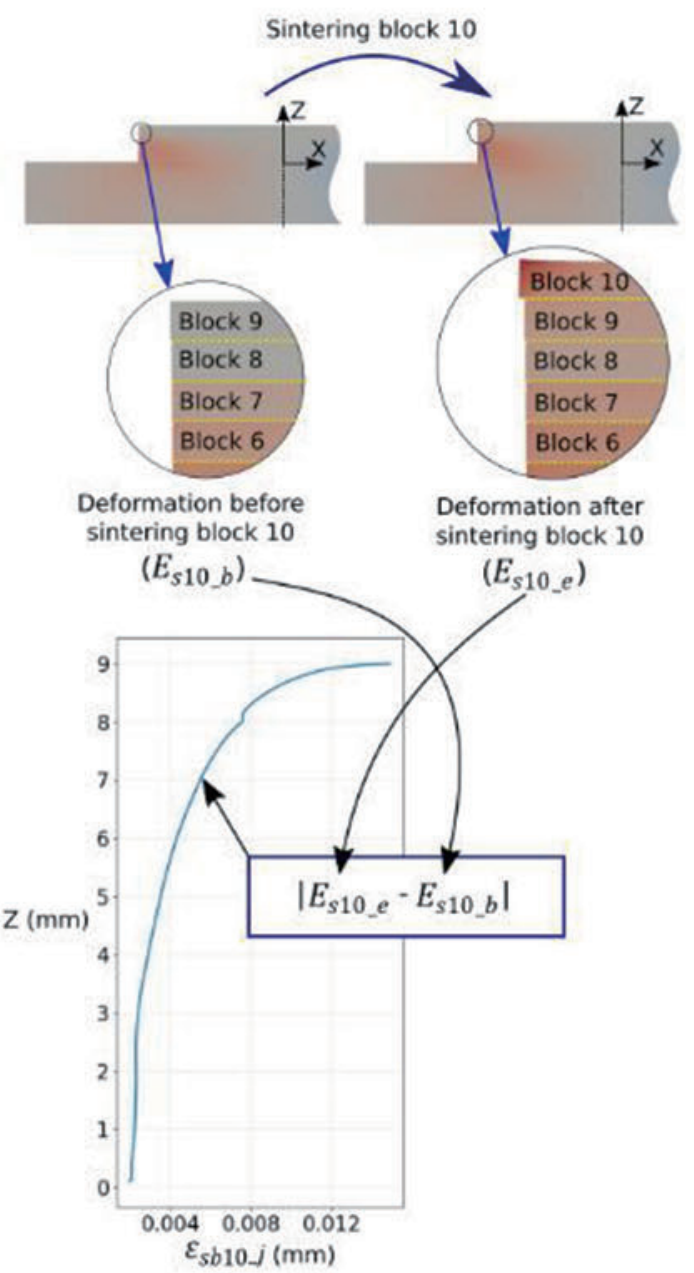

Fig. 23 Determination of shrinkage error component $\varepsilon_{s b 10_{-} j}$ in the $70 \mathrm{~mm} \times 20 \mathrm{~mm}$ sample using FEA

refer to the Reference ${ }^{16)}$.

The above section explains the process of collecting data for a shrinkage error component with the example of $\varepsilon_{s b 10_{-} j}$ of the $70 \mathrm{~mm} \times$ $20 \mathrm{~mm}$ sample. This shrinkage error component corresponds with Equation (3) that has three parameters $s_{a}, s_{b}$, and $s_{c}$. Next, $s_{a}, s_{b}$, and $s_{c}$ are interpolated with the data of $\varepsilon_{s b 10_{-j}}$ collected above. The 


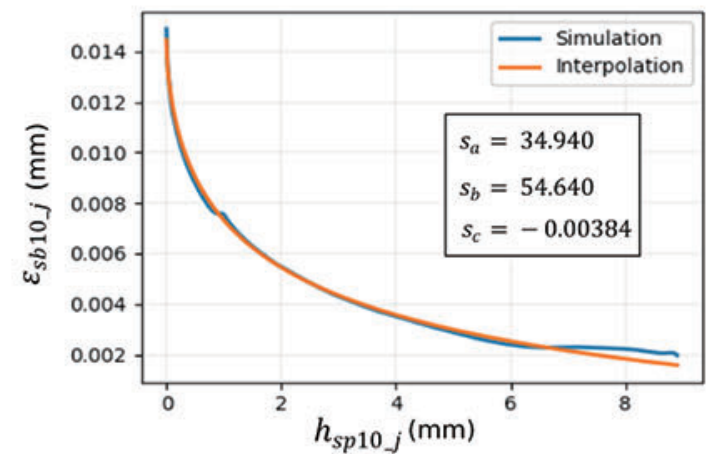

Fig. 24 Interpolation of $s_{a}, s_{b}$ and $s_{c}$ using the data of $\varepsilon_{s b 10_{-} j}$ collected by FEA in the $70 \mathrm{~mm} \times 20 \mathrm{~mm}$ sample

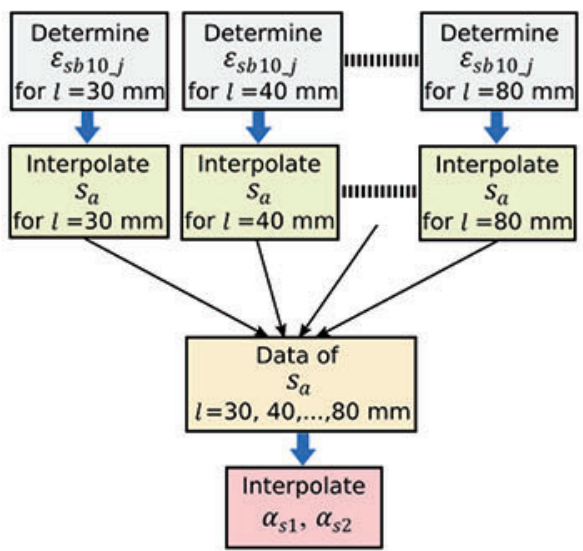

Fig. 25 Process of determining $\alpha_{s 1}$ and $\alpha_{s 2}$

interpolation results of $s_{a}, s_{b}$, and $s_{c}$ for $\varepsilon_{s b 10 j}$ are alternatively $34.94,54.640$, and -0.00384 as illustrated in Fig. 24. These values are replaced in Equation (3) to create the matched equation for the data of $\varepsilon_{s b 10_{-} j}$ as illustrated by the orange graph in Fig. 24. Comparing the result of FEA simulation and Equation (3), it shows that the difference is very small.

\subsection{Determination of parameters dependent on forming conditions}

In the proposed equations, a total of 27 parameters dependent on forming conditions are used, nine parameters to calculate the $e_{s}$ component, seven parameters to calculate the $e_{e x}$ component and 11 parameters to calculate the $e_{u c}$ component. The first two of the nine parameters are used to determine $e_{s}$ are $\alpha_{s 1}$ and $\alpha_{s 2}$ in Equation (4). The process of determining these two parameters is illustrated in Fig. 25. First, from the data of $\varepsilon_{s b 10_{-} j}$, the value of $s_{a}$ is interpolated for the corresponding value of the length $(l)$. The example in Sections 4.2 and 4.3 illustrate for the process of determination for $s_{a}$ corresponding with the $70 \mathrm{~mm} \times 20 \mathrm{~mm}$ sample $(l=70$ $\mathrm{mm})$. This process is repeated for the other samples $(l=30,40$, $\ldots, 80 \mathrm{~mm}$ ) to get the data set of $s_{a}$ which is given in Table 3 . Form this data, the same interpolation technique in Section 4.3 is applied for Equation (4) to interpolate $\alpha_{s 1}$ and $\alpha_{s 2}$. The interpolation result is given in Fig. 26, with the blue points representing the data of $s_{a}$ in Table 3, the interpolation result of $\alpha_{s 1}$ and $\alpha_{s 2}$ are respectively 214.333 and 32.405 . After interpolating $\alpha_{s 1}$ and $\alpha_{s 2}$, these values are replaced in Equation (4) and create the final equation for $s_{a}$. The diagram of this
Table 3 Interpolated values of $s_{a}$ and $s_{b}$

\begin{tabular}{ccccccc}
\hline$l(\mathrm{~mm})$ & 30 & 40 & 50 & 60 & 70 & 80 \\
\hline$s_{a}$ & 65.590 & 49.911 & 42.152 & 37.720 & 34.940 & 33.885 \\
\hline$s_{b}$ & 62.131 & 58.880 & 56.761 & 55.451 & 54.640 & 54.102 \\
\hline
\end{tabular}

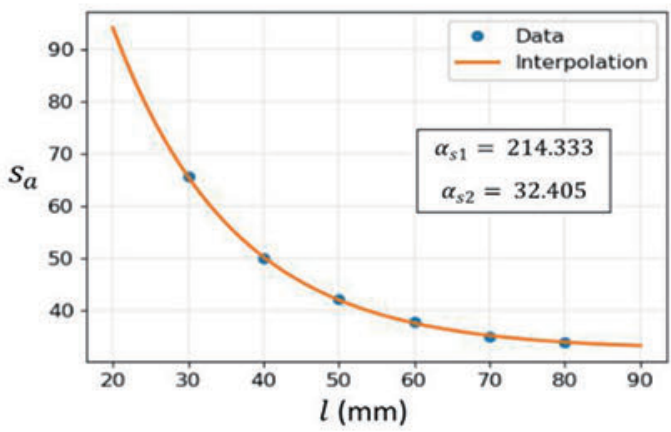

Fig. 26 Interpolation of $\alpha_{s 1}, \alpha_{s 2}$ from the calculated result of $s_{a}$

equation is illustrated by the orange graph in Fig. 26. Similarly, the other parameters used to calculate $e_{s}$ are determined and shown in Table 6.

The seven parameters used to calculate $e_{e x}$ are determined similar to those of $e_{s}$. First, the simulation data are used to interpolate the values of $s_{e a}, s_{e b}$, and $s_{e c}$ in Equation (9) corresponding with the values of $l$. The interpolation result of the $70 \mathrm{~mm} \times 20 \mathrm{~mm}$ sample corresponding with $l=70 \mathrm{~mm}$ is illustrated in Fig. 27. The interpolation results for $s_{e a}, s_{e b}$, and $s_{e c}$ of the other samples are given in Table 4.

From the results of $s_{e a}$, the parameters $\alpha_{e x 1}$ and $\alpha_{e x 2}$ of Equation (10) are interpolated. The interpolation results of $\alpha_{\text {ex } 1}$ $=483.476$ and $\alpha_{e x 2}=57.855$ are illustrated in Fig. 28. The other parameters used to calculate $e_{e x}$ are similarly determined and given in Table 6.

The 11 parameters used to calculate $e_{u c}$ are also determined similar to those of $e_{s}$ and $e_{e x}$. First, the simulation data are used to interpolate the values of $s_{u a}, s_{u b}, s_{u c}$, and $s_{u d}$ in Equation (13). The interpolation of the $70 \mathrm{~mm} \times 20 \mathrm{~mm}$ sample is illustrated in Fig. 29; the interpolation results of the other samples are given in Table 5 .

From the results of $s_{u a}$, the parameters $\alpha_{u c 1}$ and $\alpha_{u c 2}$ in Equation (14) are interpolated and illustrated in Fig. 30. The other parameters used to calculate $e_{u c}$ are similarly determined and given in Table 6.

After determining all 27 parameters, the Equations (1), (2), ..., (17) are used to calculate shrinkage error. The result of calculation for shrinkage error of the $70 \mathrm{~mm} \times 20 \mathrm{~mm}$ sample is given in Fig. 31. It shows that the result of the proposed equations and the FEA data are almost identical.

One important point needed to discuss the interpolation technique is that this technique can give more than one result. For example, in Fig. 26, the values of $\alpha_{s 1}=214.333$, and $\alpha_{s 2}=32.405$ are not the only one which is satisfied Equation (4), but there are more set values 


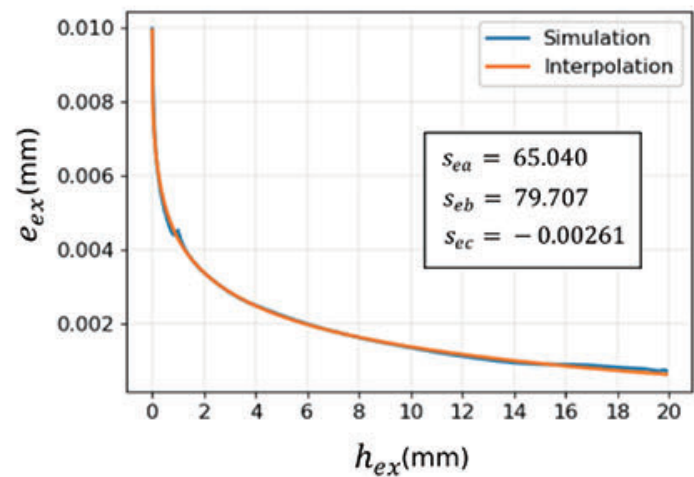

Fig. 27 Interpolation results of $s_{e a}, s_{e b}$ and $s_{e c}$ for the $70 \mathrm{~mm}$ $\times 20 \mathrm{~mm}$ sample

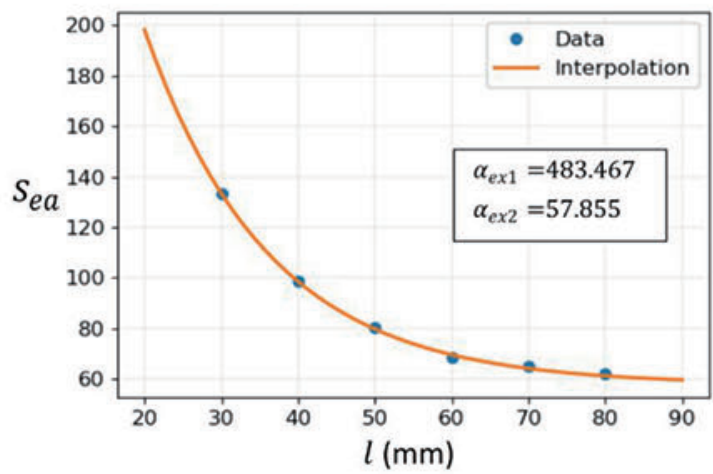

Fig. 28 Interpolation of $\alpha_{e x 1}, \alpha_{e x 2}$ from the calculated result of $s_{e a}$

Table 4 Interpolated values of $s_{e a}, s_{e b}$, and $s_{e c}$

\begin{tabular}{ccccccc}
\hline $\begin{array}{c}l \\
(\mathrm{~mm})\end{array}$ & 30 & 40 & 50 & 60 & 70 & 80 \\
\hline$s_{e a}$ & 133.565 & 98.986 & 80.239 & 68.479 & 65.040 & 62.011 \\
\hline$s_{e b}$ & 88.735 & 84.712 & 82.204 & 80.670 & 79.707 & 79.031 \\
\hline$s_{e c}$ & -0.00196 & -0.00228 & -0.00250 & -0.00259 & -0.00261 & -0.00250 \\
\hline
\end{tabular}

of $\alpha_{s 1}$ and $\alpha_{s 2}$ that can give the same result (the orange graph in Fig. 26). In this case, many sets need sampling of interpolation results to find a satisfactory one, which is the set of parameters that can be used to calculate shrinkage error that satisfy the requirement. In this research, to guarantee the reliability of the equations, experimental results are used to evaluate the calculation results. The evaluation process is presented in Section 5 .

\section{Experiments and results}

In this study, two experiments are conducted on the OPM250L machine of Sodick Co., Ltd to evaluate our mathematical model. Technical information of this machine is given in Reference ${ }^{17}$. The first experiment is performed without compensation and the second with compensation. The length of the testing sample is $l=45 \mathrm{~mm}$, and the height is $h=25 \mathrm{~mm}$. The forming condition in the experiment is given in Section 4.1. The shrinkage errors calculated from the proposed equations and the shrinkage errors of the real sample of the first experiment are given in Section 5.1. The proposed compensation technique and results of the second experiment are given in Section 5.2.

5.1 Forming without compensation

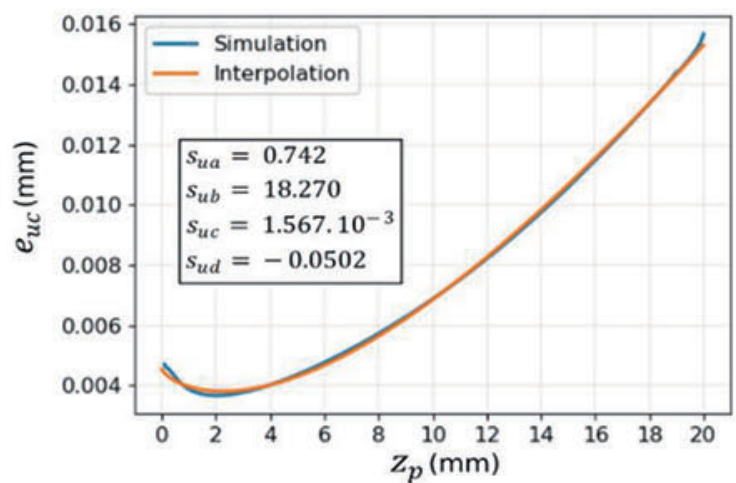

Fig. 29 Interpolation results of $s_{u a}, s_{u b}, s_{u c}$, and $s_{u d}$ for the $70 \mathrm{~mm} \times 20 \mathrm{~mm}$ sample

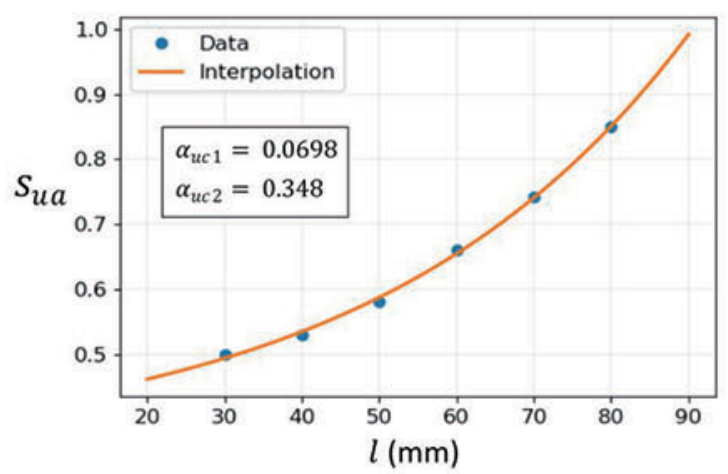

Fig. 30 Interpolation of $\alpha_{u c 1}$ and $\alpha_{u c 2}$ from the calculated result of $s_{u a}$

Table 5 Interpolated values of $s_{u a}, s_{u b}, s_{u c}$, and $s_{u d}$

\begin{tabular}{ccccccc}
\hline $\begin{array}{c}l \\
(\mathrm{~mm})\end{array}$ & 30 & 40 & 50 & 60 & 70 & 80 \\
\hline$s_{u a}$ & 0.500 & 0.530 & 0.581 & 0.660 & 0.742 & 0.850 \\
\hline$s_{u b}$ & 19.640 & 18.771 & 18.211 & 18.139 & 18.270 & 18.800 \\
\hline$s_{u c}$ & $4.77 .10^{-4}$ & $5.84 .10^{-4}$ & $8.63 .10^{-4}$ & $1.22 .10^{-3}$ & $1.57 .10^{-3}$ & $1.88 .10^{-3}$ \\
\hline$s_{u d}$ & -0.0476 & -0.0490 & -0.0504 & -0.0508 & -0.0502 & -0.0480 \\
\hline
\end{tabular}

The first experiment (forming without compensation) is used to confirm the effectiveness of shrinkage error equations. The shrinkage errors taken from the real sample of the first experiment are compared with the shrinkage errors calculated by the mathematical model. The real sample from Experiment 1 is shown in Fig. 32. After fabrication, the sample is measured using a coordinate measuring machine (CMM) CRYSTA-APEX S776 of Mitutoyo Co., Ltd that has the resolution of 0.1 $\mu \mathrm{m}$. The CMM probe head used to measure has the radius of $2 \mathrm{~mm}$, and the measuring step is $0.01 \mathrm{~mm}$. The measuring path is at the middle of the sample as illustrated in Fig. 32. At the bottom of the real part, there is a small area that could not be finished by milling (Fig. 33). Therefore, the measured data on this area cannot be used for analysis. The measured data from the bottom of the part to $\mathrm{z}=2 \mathrm{~mm}$ are ignored.

The results from the mathematical model and the experimental results are shown in Fig. 34; the shrinkage errors at the middle of each block are given in Table 7. The maximum shrinkage error on the left wall of the real sample is $0.027 \mathrm{~mm}$, at $\mathrm{z}=5.86 \mathrm{~mm}$ and the maximum shrinkage error on the right wall is $0.03 \mathrm{~mm}$, at $\mathrm{z}=3.74 \mathrm{~mm}$. Calculation error $\left(\varepsilon_{c l}\right)$ is used to evaluate the accuracy of the proposed mathematical model. The calculation error $\left(\varepsilon_{c l}\right)$ at a specific coordinate on the $z$-axis is defined as 
Table 6 Parameters for the specific forming condition

\begin{tabular}{llllll}
\hline Parameters & for & $\alpha_{s 1}=214.333$ & $\beta_{s 1}=35.208$ & $c_{s a 1}=0.0207$ & $c_{s b 1}=4.693 .10^{-7}$ \\
calculating & $e_{s j}$ & $\alpha_{s 2}=32.405$ & $\beta_{s 2}=53.186$ & $c_{s a 2}=-0.00916$ & $c_{s b 2}=-6.444 .10^{-5}$ \\
& & & & $c_{s b 3}=-0.00115$ \\
\hline Parameters & for & $\alpha_{e x 1}=483.467$ & $\beta_{e x 1}=44.539$ & $\gamma_{e x 1}=5.264 .10^{-7}$ & \\
calculating & $e_{e x}$ & $\alpha_{e x 2}=57.855$ & $\beta_{e x 2}=78.149$ & $\gamma_{e x 2}=-6.871 .10^{-5}$ & $\gamma_{e x 3}=-3.73 .10-4$ \\
\hline Parameters & for & $\alpha_{u c 1}=0.0698$ & $\beta_{u c 1}=1.743 .10^{-3}$ & $\gamma_{u c 1}=1.825 .10^{5}$ & $\delta_{u c 1}=4.643 .10^{-6}$ \\
calculating & $e_{u c}$ & $\alpha_{u c 2}=0.348$ & $\beta_{u c 2}=-0.208$ & $\gamma_{u c 2}=581.433$ & $\delta_{u c 2}=-5.279 .10^{-4}$ \\
& & & $\beta_{u c 3}=24.309$ & $\gamma_{u c 3}=3.891 .10^{-4}$ & $\delta_{u c 3}=-0.00357$ \\
\hline
\end{tabular}

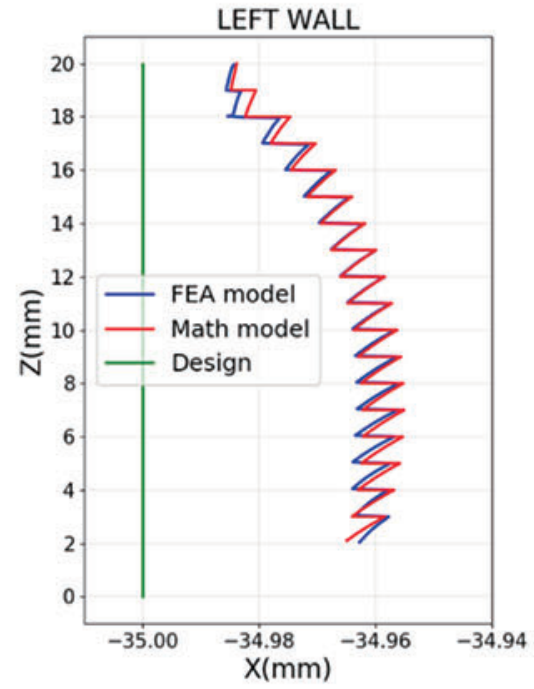

Fig. 31 Shrinkage error of the $70 \mathrm{~mm} \times 20 \mathrm{~mm}$ sample calculated using FEA and experience equation

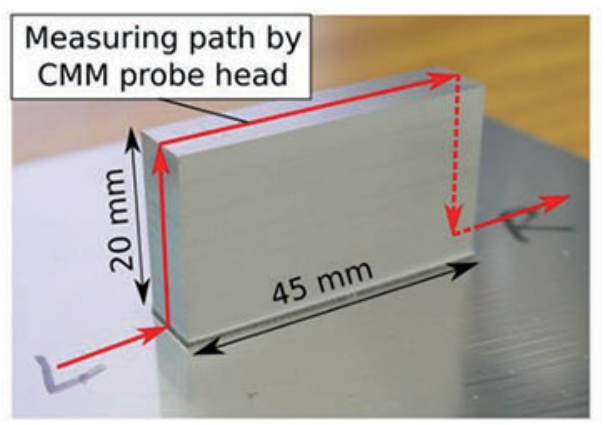

Fig. 32 Measuring path on the real sample using CMM

the difference between the calculation result and the experiment result at the coordinate. $\varepsilon_{c l}(\mathrm{~mm})$ is given by Equation (18):

$$
\varepsilon_{c l}=\left|E_{s_{-} c l}-E_{e_{-} e x}\right|,
$$

where $E_{s_{-} c l}(\mathrm{~mm})$ is the shrinkage error calculated from the mathematical model, $E_{e_{-} e x}(\mathrm{~mm})$ is the shrinkage error from the experiment. The values at the middle of each block are used to analyze the calculation error. The calculation errors at the middle of all blocks are given in Table 7. The average of $\varepsilon_{c l}$ is $0.0037 \mathrm{~mm}$, with a standard deviation of $0.0021 \mathrm{~mm}$.

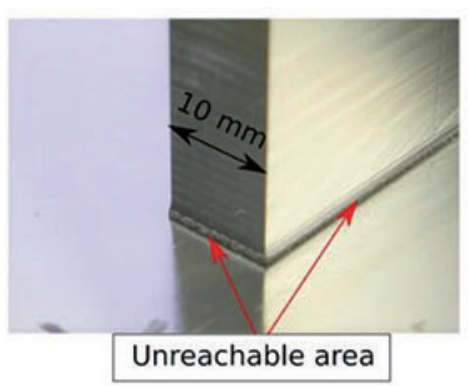

Fig. 33 Area at the bottom of the real part
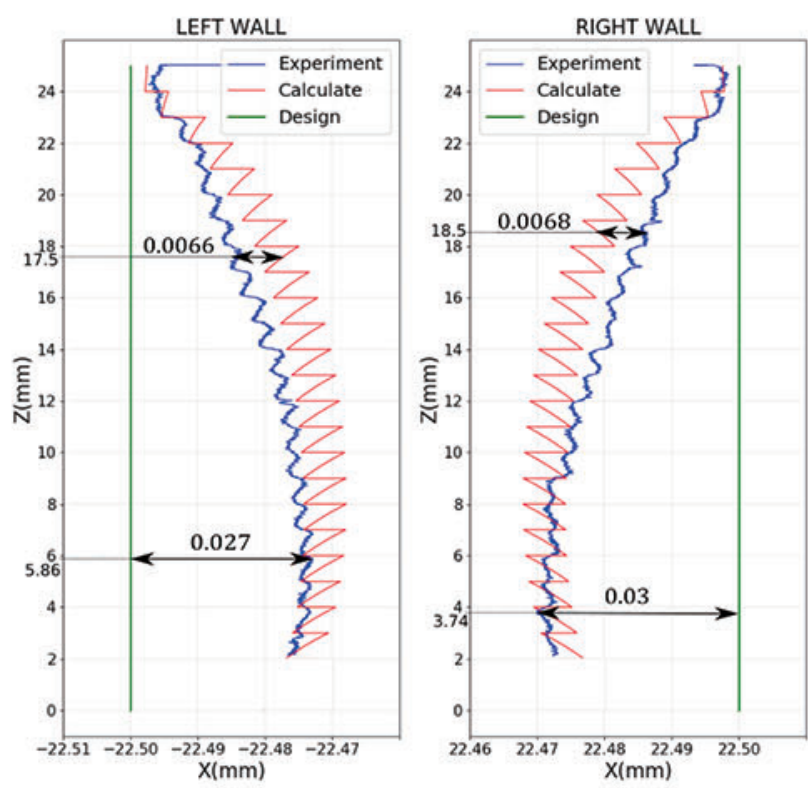

Fig. 34 Results of calculation and experiment without compensation

The maximum $\varepsilon_{c l}$ on the left wall is $0.0066 \mathrm{~mm}$ at Block 17, and the maximum $\varepsilon_{c l}$ on the right wall is $0.0068 \mathrm{~mm}$ at Block 18 .

Experimental results have a specific measuring error, which is explained as follows. The CMM probe head is a ball; therefore, it cannot measure the corners at less than $180^{\circ}$. This measurement error cannot be avoided, and is illustrated in Fig. 35. Fortunately, this measurement error only affects the top of blocks, the measuring results from the middle to the bottom are still satisfactory for analysis.

\subsection{Forming with compensation}


Table 7 Shrinkage errors without compensation at the middle of the blocks

\begin{tabular}{c|c|c|c|c|c|c}
\hline $\mathbf{Z}(\mathbf{m}$ & \multicolumn{3}{|c|}{ Left wall } & \multicolumn{3}{c}{ Right wall } \\
\cline { 2 - 7 } $\mathrm{m})$ & $\begin{array}{c}E_{s_{-} c l} \\
(\mathrm{~mm})\end{array}$ & $\begin{array}{c}E_{e_{e} e x} \\
(\mathrm{~mm})\end{array}$ & $\begin{array}{c}\varepsilon_{c l} \\
(\mathrm{~mm})\end{array}$ & $\begin{array}{c}E_{s_{-} c l} \\
(\mathrm{~mm})\end{array}$ & $\begin{array}{c}E_{s_{-} e x} \\
(\mathrm{~mm})\end{array}$ & $\begin{array}{c}\varepsilon_{c l} \\
(\mathrm{~mm})\end{array}$ \\
\hline 2.5 & 0.0259 & 0.0247 & 0.0012 & 0.0259 & 0.0277 & 0.0018 \\
\hline 3.5 & 0.0270 & 0.0254 & 0.0016 & 0.0270 & 0.0286 & 0.0016 \\
\hline 4.5 & 0.0277 & 0.0256 & 0.0021 & 0.0277 & 0.0275 & 0.0002 \\
\hline 5.5 & 0.0282 & 0.0261 & 0.0021 & 0.0282 & 0.0285 & 0.0003 \\
\hline 6.5 & 0.0285 & 0.0259 & 0.0026 & 0.0285 & 0.0269 & 0.0016 \\
\hline 7.5 & 0.0286 & 0.0250 & 0.0036 & 0.0286 & 0.0277 & 0.0009 \\
\hline 8.5 & 0.0286 & 0.0248 & 0.0038 & 0.0286 & 0.0280 & 0.0006 \\
\hline 9.5 & 0.0285 & 0.0235 & 0.0050 & 0.0285 & 0.0254 & 0.0031 \\
\hline 10.5 & 0.0282 & 0.0237 & 0.0045 & 0.0282 & 0.0248 & 0.0034 \\
\hline 11.5 & 0.0278 & 0.0233 & 0.0045 & 0.0278 & 0.0243 & 0.0035 \\
\hline 12.5 & 0.0273 & 0.0225 & 0.0048 & 0.0273 & 0.0227 & 0.0046 \\
\hline 13.5 & 0.0266 & 0.0220 & 0.0046 & 0.0266 & 0.0214 & 0.0052 \\
\hline 14.5 & 0.0258 & 0.0200 & 0.0058 & 0.0258 & 0.0191 & 0.0067 \\
\hline 15.5 & 0.0248 & 0.0186 & 0.0062 & 0.0248 & 0.0186 & 0.0062 \\
\hline 16.5 & 0.0236 & 0.0174 & 0.0062 & 0.0236 & 0.0174 & 0.0062 \\
\hline 17.5 & 0.0222 & 0.0156 & 0.0066 & 0.0222 & 0.0156 & 0.0066 \\
\hline 18.5 & 0.0206 & 0.0144 & 0.0062 & 0.0206 & 0.0138 & 0.0068 \\
\hline 19.5 & 0.0186 & 0.0130 & 0.0056 & 0.0186 & 0.0126 & 0.0060 \\
\hline 20.5 & 0.0162 & 0.0113 & 0.0049 & 0.0162 & 0.0108 & 0.0054 \\
\hline 21.5 & 0.0133 & 0.0102 & 0.0031 & 0.0133 & 0.0093 & 0.0040 \\
\hline 22.5 & 0.0097 & 0.0073 & 0.0024 & 0.0097 & 0.0061 & 0.0036 \\
\hline 23.5 & 0.0050 & 0.0039 & 0.0011 & 0.0050 & 0.0028 & 0.0022 \\
\hline 24.5 & 0.0023 & 0.0034 & 0.0011 & 0.0023 & 0.0022 & 0.0001 \\
\hline & & & & & & \\
\hline
\end{tabular}

Based on the results of the mathematical model, a compensation technique is proposed to reduce SHM shrinkage error. In the forming process without compensation, the setting for finishing milling is taken from the model design. For example, in the first experiment, the finishing milling setting on the $\mathrm{x}$-axis of the right wall is $22.5 \mathrm{~mm}$ for all the blocks. In our compensation technique, the shrinkage errors calculated from the mathematical model $\left(E_{s_{-} c l}\right)$ are added to the finishing milling setting. Consequently, it is expected that after shrinking, the dimensions of the sample, to which compensation values were added, would have the exact values as the initial design. The compensation settings $\left(D_{c}\right)$ for finishing milling on the right wall of the sample are given by Equation (19):

$$
D_{c}=D_{d}+E_{s_{-} c l},
$$

where $D_{c}(\mathrm{~mm})$ is the compensation setting, $D_{d}(\mathrm{~mm})$ is the initial setting for finishing milling based on the designed model, and $E_{s_{-} c l}$ is given in Table 5, as explained above. In this study, the model is symmetric; therefore, the milling settings for the left wall are similar to the settings on the right wall but negative. The compensation setting values in the second experiment are given in Table 8, and illustrated in Fig. 36.

After forming, the sample in the second experiment is also measured on CMM the same as in the first experiment. The results of the second experiment are shown in Fig. 36. The maximum shrinkage error on the left wall is $0.0074 \mathrm{~mm}$ at $\mathrm{z}=16.12 \mathrm{~mm}$ and the maximum shrinkage error on the right wall is $0.008 \mathrm{~mm}$ at $\mathrm{z}=14.06 \mathrm{~mm}$.

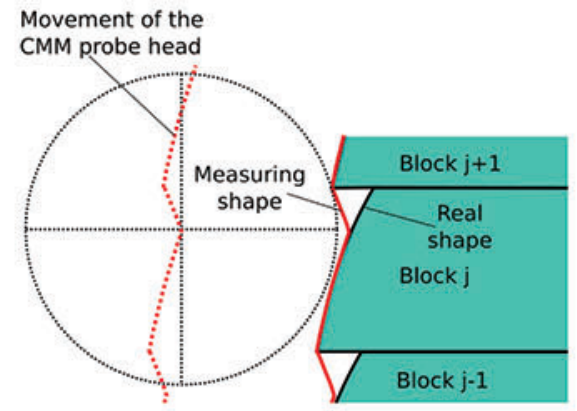

Fig. 35 Measuring error due to the CMM probe head

Table 8 Compensation setting for finishing milling on the right wall

\begin{tabular}{c|c|c|c|c|c}
\hline Block & $\begin{array}{c}\text { Setting } \\
(\mathbf{m m})\end{array}$ & Block & $\begin{array}{c}\text { Setting } \\
(\mathbf{m m})\end{array}$ & Block & $\begin{array}{c}\text { Setting } \\
(\mathbf{m m})\end{array}$ \\
\hline 1 & 22.523 & 10 & 22.529 & 19 & 22.521 \\
\hline 2 & 22.525 & 11 & 22.528 & 20 & 22.519 \\
\hline 3 & 22.526 & 12 & 22.528 & 21 & 22.516 \\
\hline 4 & 22.527 & 13 & 22.527 & 22 & 22.513 \\
\hline 5 & 22.528 & 14 & 22.527 & 23 & 22.510 \\
\hline 6 & 22.528 & 15 & 22.526 & 24 & 22.505 \\
\hline 7 & 22.529 & 16 & 22.525 & 25 & 22.502 \\
\hline 8 & 22.529 & 17 & 22.524 & & \\
\hline 9 & 22.529 & 18 & 22.522 & & \\
\hline
\end{tabular}

\section{Discussion}

\subsection{Evaluation of the shrinkage error equations for SHM}

First, the result of the first experiment (Fig. 34) shows that the shape of the shrinkage error in the real sample and the result of the calculation are similar. In general, the shrinkage error has a saw-tooth shape, and shrinkage errors at the lower blocks are larger than the higher blocks. Shrinkage error below Block 10 becomes stable due to the distance to the shrinking layers being large enough. Moreover, in one block, the top of the block deforms more than the bottom. This result satisfies the mechanism of shrinkage error presented in Section 2.

However, Fig. 34 also shows a small asymmetric error in the experimental result. This study used a $2 \mathrm{D}$ model which assumes that the cooling process and shrinkage are absolutely uniform. It also means that the asymmetric error is assumed to be zero. However, in the actual fabricating process, the shrinkage is three-dimensional (3D) phenomena, and not as perfectly symmetric as the 2D model. As shown in Fig. 18, a laser beam is asymmetrically and three-dimensionally scanned on the metal powder from the right to the left of the part. Therefore, the right side of the part cools before cooling of the left side; as the result, the part is deformed to the right side. This explanation matches the asymmetric error phenomenon in Fig. 34. However, Fig. 34 also shows that this error is very small compared with the maximum shrinkage error. If the asymmetric error is considered, 3D models which take significantly longer time for FEA must be used. However, the improvement of using $3 \mathrm{D}$ models is very limited. This is the reason why the 2D model is used in this study.

The accuracy of the calculation is very important because it decides the accuracy improvement of the compensation technique. The calculation error $\left(\varepsilon_{c l}\right)$ in Table 7 is used to evaluate the accuracy of the shrinkage error 

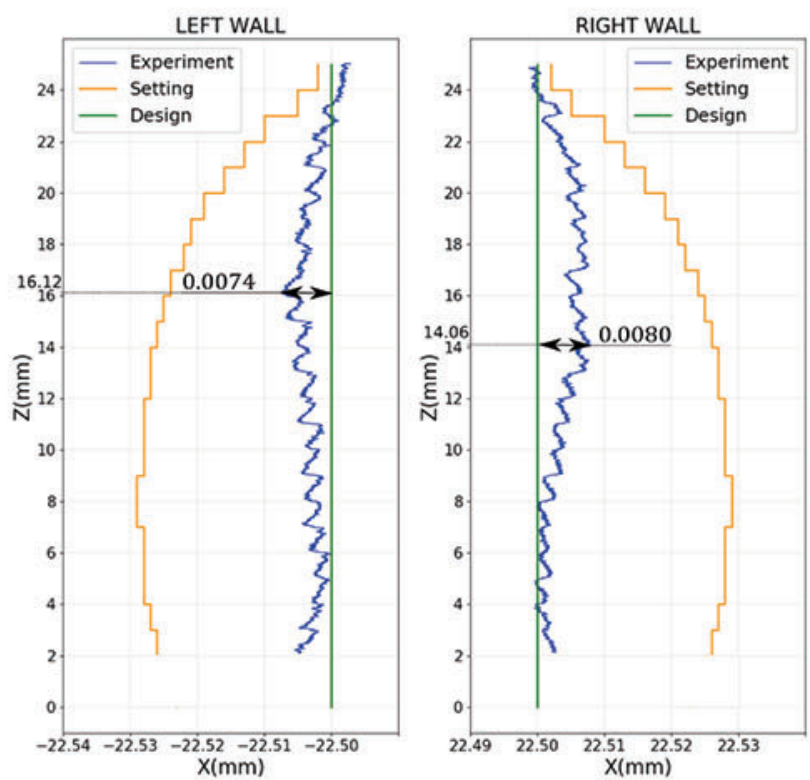

Fig. 36 Setting for finishing milling with compensation and results of the experiment

equations. In the first experiment, the mean of $\varepsilon_{c l}$ is $0.0037 \mathrm{~mm}$, with a standard deviation of $0.0021 \mathrm{~mm}$. The maximum $\varepsilon_{c l}$ on the left wall is $0.0066 \mathrm{~mm}$ at Block 17 and the maximum $\varepsilon_{c l}$ on the right wall is 0.0068 $\mathrm{mm}$ at Block 18. Compared with the maximum shrinkage error in the first experiment $(0.03 \mathrm{~mm})$, the calculation error is very small and satisfies the accuracy requirement of $0.01 \mathrm{~mm}$. In this study, $0.01 \mathrm{~mm}$ is set to be the required accuracy based on the accuracy of the sample products in Reference ${ }^{17}$. This is the expectation of manufacturers in industry; therefore, satisfying this accuracy confirms the potential of the proposed method in actual manufacturing. The $0.01 \mathrm{~mm}$ error is only used to evaluate the effectiveness of the method, and is not used in any calculation.

The time taken for calculation is another advantage of the mathematical model. As mentioned above, in the previous study, FEA is used to calculated shrinkage error. The FEA limitation is time-consuming. For the sample of the same size as the sample in this study, FEA would take three to four days to calculate the shrinkage error. Hence, it is not suitable for actual production. Our proposed mathematical model completely solves this problem by taking only a few seconds to calculate a set of shrinkage errors as seen in Fig. 34.

The objective of introducing these equations is to replace FEA processes for estimating shrinkage error. FEA is used to estimate the shrinkage errors of 6 samples corresponding with the part length $1=30$ $\mathrm{mm}, 40 \mathrm{~mm}, 50 \mathrm{~mm}, 60 \mathrm{~mm}, 70 \mathrm{~mm}$, and $80 \mathrm{~mm}$. The FEA shrinkage error results of the 6 samples are used to obtain the interpolation equations (3) - (17). With these equations, the shrinkage error of any part length $(l)$ from $30 \mathrm{~mm}$ to $80 \mathrm{~mm}$ (e.g. $l=33.5 \mathrm{~mm}, 47.1 \mathrm{~mm}, 69.9 \mathrm{~mm}, \ldots$ ) can be directly calculated while FEA takes long hours to calculate it. The proposed method using the equations has significant advantage than the FEA method in term of calculation time. This advantage is extremely considerable in practical application.

\subsection{Efficiency of compensation technique}

The efficiency of the compensation technique is the second important research issue. As mentioned above, in the proposed compensation technique, compensation settings are calculated directly from the results of the equations. Therefore, the accuracy of the equations determines the
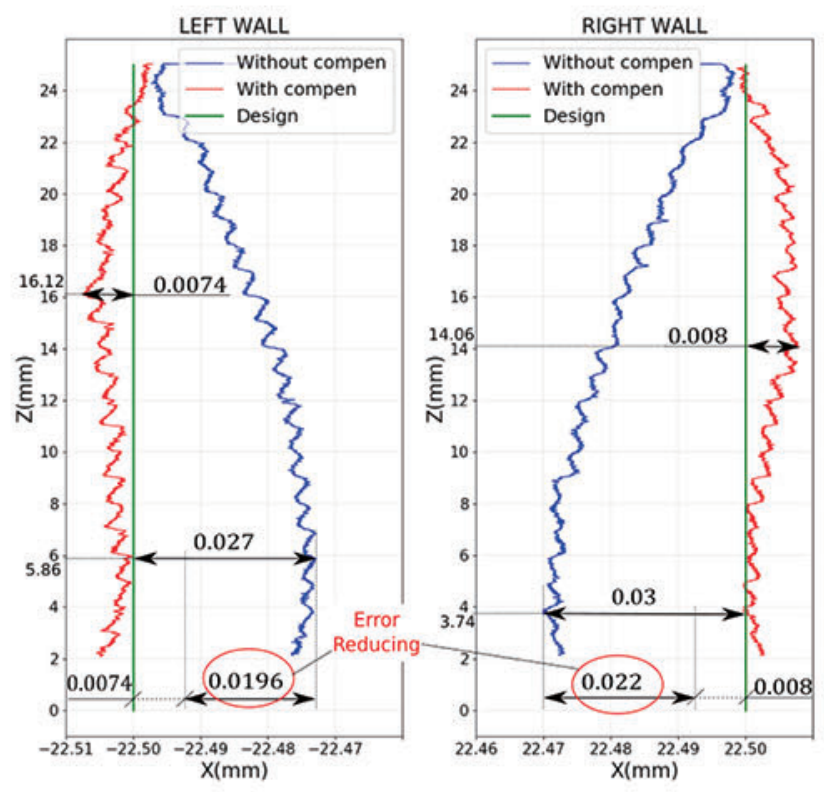

Fig. 37 Efficiency of compensation in reducing shrinkage error

accuracy of the sample. If the calculation error is less than the accuracy requirement, then the accuracy of the sample will probably meet expectations. A comparison between the samples in the first and second experiments is illustrated in Fig. 37. This shows that after applying the compensation technique, the error on the left wall reduces $0.0196 \mathrm{~mm}$ (from $0.027 \mathrm{~mm}$ to $0.0074 \mathrm{~mm}$ ) and the error on the right wall reduces $0.022 \mathrm{~mm}$ (from $0.03 \mathrm{~mm}$ to $0.008 \mathrm{~mm}$ ). This error reduction is considered a significant improvement as it proves that the proposed model and compensation technique can be applied in the actual SHM production activities.

Fig. 37 shows that the error before compensation is convex, and the error after compensation does not have a specific shape; which is explained as follows. The experimental error contains not only shrinkage error but also the others such as tool deflection, and tool wear errors. However, the shrinkage error is much larger than the others; moreover, because shrinkage error has a convex shape, the error before compensation is also convex. After compensation, the shrinkage error is almost eliminated, the error now is the combination of the others including the calculation error. However, these errors are very small in the tested forming conditions.

In general, the experiment with compensation proves that the compensation technique using the results of the proposed model can significantly reduce shrinkage error. The accuracy of the sample after compensation is significantly improved although the other errors cannot be completely eliminated.

\subsection{Limitations}

This study has the following limitations. First, in this mathematical model, there are only two input parameters, the length $(l)$ and the height ( $h$ ) of the part. The others parameters such as the part width, layer thickness, and block thickness must be investigated in future research. Next, only one specific SHM forming condition has been investigated. Before the proposed mathematical model can be used in industry, it must be investigated with a variety of forming conditions. Another limitation is that this study only studied shrinkage error in a rectangular-shaped product, which is a very simple SHM shape. With complicated shapes, the length 
and width of the sample will change, and the proposed model cannot be used. Future studies should investigate shrinkage error for other shape types. In this study, the proposed method investigates and confirms the efficiency for the part length from $30 \mathrm{~mm}$ to $80 \mathrm{~mm}$. If it is required to expand the application of the method for the larger or smaller size samples, more studies need to be conducted. If the current equations are not accurate enough, further analytical processes are required to improve the equations. In the actual manufacturing, some other phenomena could affect to the shrinkage error. If thermal treatment is required to reduce the residual stress, it is predicted that the shape of a product is changed. Alternatively, the base plate's stiffness could also affect to the shape error. These phenomena are not included in this study; therefore, there is no evidence to discuss about them.

\section{Conclusion}

This study proposes a new efficient method to calculate SHM shrinkage error with the advantage of an extremely short calculation time. This method derives a mathematical model based on the shrinkage error data collected from FEA. In this study, a mathematical model is derived for a rectangular shape with two variables: the length and height. This mathematical model is tested for a specific forming condition in SHM and gives a high accuracy result. The calculation results of this mathematical model are used to compensate and give a significant reduction of dimensional error in the sample. The dimensional error after compensation is small enough to meet the expectations of actual manufacturing. This confirms the potential of the proposed method to enhance the accuracy of SHM in industry.

\section{References}

1) H. Bikas, P. Stavropoulos, and G. Chryssolouris: Additive manufacturing methods and modeling approaches: A critical review, Int. J. Adv. Manuf. Technol., vol. 83, no. 1-4, pp. 389-405, 2016.

2) W. Du, Q. Bai, and B. Zhang: A Novel Method for Additive/Subtractive Hybrid Manufacturing of Metallic Parts, Procedia Manuf., vol. 5, pp. 1018-1030, 2016.

3) W. Du, Q. Bai, and B. Zhang: Machining characteristics of $18 \mathrm{Ni}-300$ steel in additive/subtractive hybrid manufacturing, Int. J. Adv. Manuf. Technol., vol. 95, no. 5-8, pp. 2509-2519, 2018.

4) M. Behandish, S. Nelaturi, and J. De Kleer: Computer-Aided Design Automated process planning for hybrid manufacturing, Comput. Des., vol. 102, pp. 115-127, 2018.

5) https://www.j-platpat.inpit.go.jp/c1800/PU/JP3687672/5BFF8032FAF1D1FC141F284D26A5CA81777A C0506B4AA07932FBF5F9E5989814/15/ja (Panasonic, Finishing Methods for Powder Sinking Parts) (Access: July 19, 2019).

6) C. Li, J. F. Liu, X. Y. Fang, and Y. B. Guo: Efficient predictive model of part distortion and residual stress in selective laser melting, Addit. Manuf., vol. 17, pp. 157-168, 2017.

7) M. F. Zaeh and G. Branner: Investigations on residual stresses and deformation in selective laser melting, Prod,
Eng., vol. 4, no. 1, pp. 35-45, 2010.

8) J. P. Kruth, J. Deckers, E. Yasa, and R. Wauthlé: Assessing and comparing influencing factors of residual stresses in selective laser melting using a novel analysis method, Proc. Inst. Mech. Eng. Part B J. Eng. Manuf., vol. 226, no. 6, pp. 980-991, 2012.

9) N.T. Nghiep, H. Aoyama and I. Araie: High-precision Forming Process Based on Analysis of Selective Laser Sintering Hybrid Milling, J. Jpn. Soc. Precis. Eng., accepted (2020).

10) https://www.tokkin.com/materials/special_steel/carbon_stee 1 (Tokkin, Carbon Steels (S15C, S45C, S50C, S55C, \& S60C)) (Access: July 19, 2019).

11) https://www.tokkin.com/materials/high_parformance/mas1 (Tokkin, Maraging Steels (MAS-1)) (Access: July 19, 2019).

12) Mahamood R.M. Laser Basics and Laser Material Interactions. In: Laser Metal Deposition Process of Metals, Alloys, and Composite Materials. Engineering Materials and Processes. Springer, Cham (2018).

13) https://www.code-aster.org/V2/doc/default/en/man_r $r / \mathrm{r} 5 / \mathrm{r} 5.0$ 3.02.pdf (Integration of the relations of behavior elastoplastic of Von Mises).

14) “ scipy.optimize.curve_fit - SciPy v1.3.1 Reference Guide." [Online]. Available: https://docs.scipy.org/doc/scipy/reference/generated/scipy.optimize. curve_fit.html [Accessed: 23-Oct-2019].

15) Rokem: A short course about fitting models with the scipy.optimize module, Journal of Open Source Education, 1(2), 16, 2018.

16) Young P., Fitting Data to a Model. In: Everything You Wanted to Know About Data Analysis and Fitting but Were Afraid to Ask. SpringerBriefs in Physics. Springer, Cham, 2015.

17) https://www.sodick.com/products/metal-3dprinting/opm2501 (Sodick, Metal 3D Printer OPM250L) (Access: July 19, 2019). 